\title{
How to Accurately and Privately Identify Anomalies
}

\author{
Hafiz Asif \\ Rutgers University \\ hafiz.asif@rutgers.edu
}

\author{
Periklis A. Papakonstantinou \\ Rutgers University \\ periklis.research@gmail.com
}

\author{
Jaideep Vaidya \\ Rutgers University \\ jsvaidya@business.rutgers.edu
}

\begin{abstract}
Identifying anomalies in data is central to the advancement of science, national security, and finance. However, privacy concerns restrict our ability to analyze data. Can we lift these restrictions and accurately identify anomalies without hurting the privacy of those who contribute their data? We address this question for the most practically relevant case, where a record is considered anomalous relative to other records.

We make four contributions. First, we introduce the notion of sensitive privacy, which conceptualizes what it means to privately identify anomalies. Sensitive privacy generalizes the important concept of differential privacy and is amenable to analysis. Importantly, sensitive privacy admits algorithmic constructions that provide strong and practically meaningful privacy and utility guarantees. Second, we show that differential privacy is inherently incapable of accurately and privately identifying anomalies; in this sense, our generalization is necessary. Third, we provide a general compiler that takes as input a differentially private mechanism (which has bad utility for anomaly identification) and transforms it into a sensitively private one. This compiler, which is mostly of theoretical importance, is shown to output a mechanism whose utility greatly improves over the utility of the input mechanism. As our fourth contribution we propose mechanisms for a popular definition of anomaly $((\beta, r)$-anomaly) that (i) are guaranteed to be sensitively private, (ii) come with provable utility guarantees, and (iii) are empirically shown to have an overwhelmingly accurate performance over a range of datasets and evaluation criteria.
\end{abstract}

\section{CCS CONCEPTS}

-Security and privacy $\rightarrow$ Privacy-preserving protocols; • Computing methodologies $\rightarrow$ Anomaly detection.

\section{KEYWORDS}

privacy; anomaly identification; differential privacy; outlier detection

\section{ACM Reference Format:}

Hafiz Asif, Periklis A. Papakonstantinou, and Jaideep Vaidya. 2019. How to Accurately and Privately Identify Anomalies. In 2019 ACM SIGSAC Conference on Computer and Communications Security (CCS '19), November 11-15, 2019, London, United Kingdom. ACM, New York, NY, USA, 18 pages. https://doi.org/10.1145/3319535.3363209

Permission to make digital or hard copies of all or part of this work for personal or classroom use is granted without fee provided that copies are not made or distributed for profit or commercial advantage and that copies bear this notice and the full citation on the first page. Copyrights for components of this work owned by others than ACM must be honored. Abstracting with credit is permitted. To copy otherwise, or republish, to post on servers or to redistribute to lists, requires prior specific permission and/or a fee. Request permissions from permissions@acm.org.

CCS '19, November 11-15, 2019, London, United Kingdom

(C) 2019 Association for Computing Machinery.

ACM ISBN 978-1-4503-6747-9/19/11 ..\$15.00

https://doi.org/10.1145/3319535.3363209

\section{INTRODUCTION}

At the forefront of today's research in medicine and natural sciences is the use of data analytics to discover complex patterns from vast amounts of data [11, 23, 39]. While this approach is incredibly useful, it raises serious privacy-related ethical and legal concerns $[5,7,20,21]$ because inferences can be drawn from the analysis of the person's data to the person's identity, causing a privacy breach [19, 24, 26, 27, 37]. In this work, we focus specifically on the problem of identifying anomalous records, which has fundamental applications in many domains and is also crucial for scientific advancements [1, 3, 30, 40, 42]. For example, to treat cancer, we must tell if a tumor is malignant; to stop bank fraud, we must flag the suspicious transactions; and to counter terrorism, we must identify the individuals exhibiting extreme behavior. Note that in such settings, it is imperative to accurately identify the anomalies, e.g., it is critical to identify the fraudulent transactions. However, in all these situations, it is still essential to protect the privacy of the normal (i.e., non-anomalous) records [7, 21] (e.g., customers with a legitimate transaction or patients with a benign tumor) while not sacrificing accuracy (e.g., labeling a malignant tumor as benign).

We solve the problem of accurate, private, and algorithmic anomaly identification (i.e., labeling a record as anomalous or normal by an algorithm) with an emphasis on reducing false negative labeling an anomaly as normal - rate. The current methods for protecting privacy work well for doing statistics and other aggregate tasks $[17,18]$, but they are inherently unable to identify anomalous records accurately. Furthermore, the modern methods of anomaly identification label a record as anomalous (or normal) based on its degree of dissimilarity from the other existing records $[1,3,8,35]$. Consequently, the labeling of a record as anomalous is specific to a dataset, and knowing that a record is anomalous can leak a significant amount of information about the other records. This type of privacy leakage is the core obstacle that any privacypreserving anomaly identification method must overcome. This work is the first to develop methods (in a general setting where anomalies are data-dependent) to accurately identify if a record is anomalous while simultaneously guaranteeing privacy by making it statistically impossible to infer if a non-anomalous record was included in the dataset.

We formalize a notion of privacy appropriate for anomaly detection and identification and develop general constructions to achieve this. Note that we assume a trusted curator, who performs the anomaly identification. If the data is distributed and the trusted curator is not available, one can employ secure multiparty computation to simulate the trusted curator [9], where now the same methodology as in the previous setting can be used.

Although the privacy definitions and constructions we develop are not tied to any specific anomaly definition, we instantiate them for a specific kind of anomaly: $(\beta, r)$-anomaly [35], which is a widely prevalent model for characterizing anomalies and generalizes many 


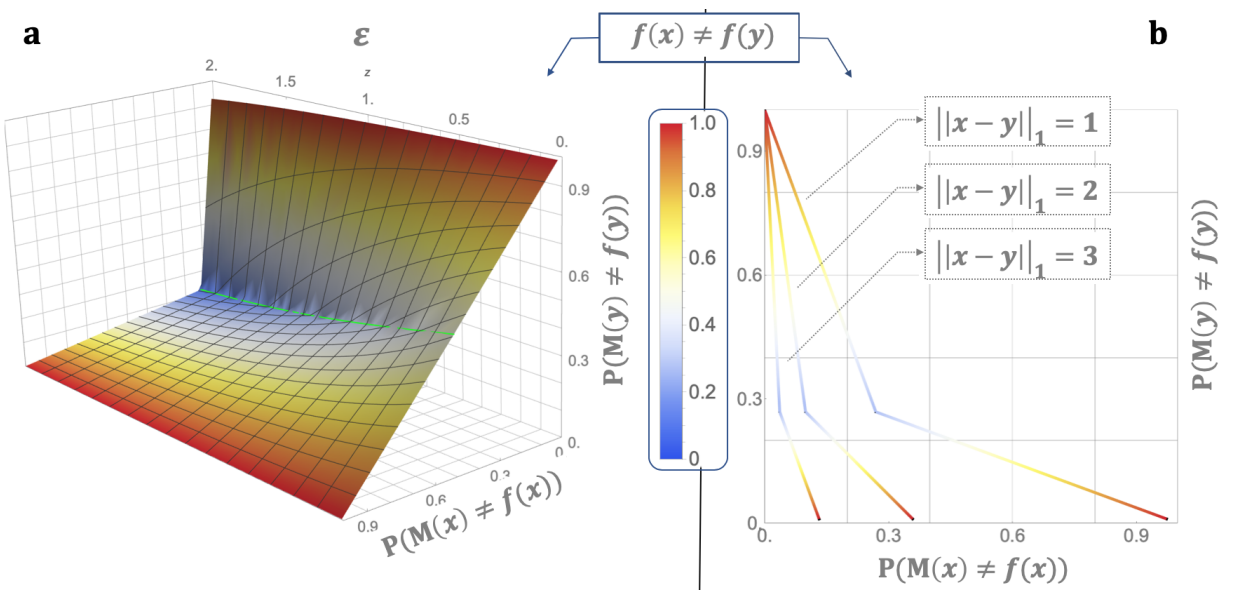

Figure 1: (a) $x$ and $y$ differ by one record, the " $\varepsilon$ axis" is for the privacy parameter, the " $P(M(x) \neq f(x))$ axis" is for the minimum error over all $\varepsilon$-DP mechanisms $M$ on $x$ for a give error on $y$ on the " $P(M(y) \neq f(y))$ axis". The graph depicts the tradeoff between the errors committed on $x$ and $y$. (b) this plot is for $\varepsilon=1$ and otherwise is the same but for different $x$ 's and $y$ 's.

other definitions of anomalies [3, 22, 34, 35]. These technical instantiations naturally extend to the other well-known variants of this formalization [1]. Under this anomaly definition, a record (which lives in a metric space) is considered anomalous if there are at most $\beta$ records similar to it, i.e., within distance $r$. The parameters $\beta$ and $r$ are given by domain experts [35] or found through exploratory analysis by possibly using differentially private methods $[17,18]$ (since these parameters can be obtained by minimizing an aggregate statistic, e.g., risk or average error) to protect privacy in this process.

\subsection{Why do we need a new privacy notion?}

We consider the trusted curator setting for the privacy. The trusted curator has access to the database, and it answers the anomaly identification queries using a mechanism. The privacy of an individual is protected if the output of an anomaly identification mechanism is unaffected by the presence or the absence of the individual's record in the database (which is the input to the mechanism). This is the notion of privacy (i.e. protection) of a record that we consider here; it protects the individual against any risk incurred due to the presence of its information and was first formalized in the seminal work of differential privacy $[15,17]$ (where privacy is quantified by a parameter $\varepsilon>0$ : the smaller the $\varepsilon$, the higher the privacy) and can informally be stated as follows: a randomized mechanism that takes a database as input is $\varepsilon$-differentially private if for any two input databases differing by one record, the probabilities (corresponding to the two databases) of occurrence of any event are within a multiplicative factor $e^{\varepsilon}$ (i.e., are almost the same in all cases). Unfortunately, simply employing differential privacy does not address the need for both privacy and practically meaningful accuracy guarantees in our case. For example, providing privacy equally to everyone severely degrades accuracy in identifying anomalies. For a database, the addition of a record in a region which is sparse in terms of data points creates an anomaly. Conversely, the removal of an anomalous record typically removes the anomaly altogether. Therefore, the accuracy achievable for anomaly identification via differential privacy is limited as explained below.
Differential privacy for binary functions $f: \mathcal{D} \rightarrow\{0,1\}$, such as the anomaly identification, comes with inherent limitations that can be explained through the graph of Figure 1a. Fix any mechanism $M$ that is supposed to compute $f$, with the property that this mechanism is differentially private. The mere fact that $f$ is binary and $M$ is differentially private has the following effect. For any two databases $x$ and $y$ that differ in one record say that $f(x)=0$ and $f(y)=1$. Now, a simple calculation shows that the differential privacy constraints create a tradeoff: whenever $M$ makes a small error in computing $f(x)$ then it is forced to err a lot when computing on its "neighbor" $y$ and vice-versa. Moreover, the higher the privacy requirements are (i.e. for smaller $\varepsilon$ ) the stricter this tradeoff is, as depicted on Figure 1a. Formally, we state this fact as follows.

Claim 1. Fix $\varepsilon>0, f: \mathcal{D} \rightarrow\{0,1\}$, and $\varepsilon-D P M: \mathcal{D} \rightarrow\{0,1\}$ arbitrarily. For every $x$ and $y$, if $f(x) \neq f(y)$ and $\|x-y\|_{1}=1$, then $P(M(x) \neq f(x)) \geq 1 /\left(1+e^{\varepsilon}\right)$ or $P(M(y) \neq f(y)) \geq 1 /\left(1+e^{\varepsilon}\right)$.

What happens to this inherent tradeoff when $x$ and $y$ differ in more than one record? As shown on Figure 1b this tradeoff is relaxed. We note that for deriving the tradeoff, there was nothing specific to the $\ell_{1}$ metric (used for differential privacy), but instead we could have used any metric over the space of databases; other works that considered general metrics are e.g., [25, 33]. Our work proposes a distance metric which is appropriate for anomaly identification, in conjunction to an appropriate relaxation of differential privacy. This way we will lay out a practically meaningful (but also amenable to analysis) privacy setting.

\subsection{What do we want from the new notion?}

We want to relax differential privacy since affording protection for everyone severely degrades the accuracy for anomaly identification. One possible relaxation, suitable for the problem at hand, is providing protection only for a subset of the records. We note that such a relaxation is backed by privacy legislation, e.g., GDPR allows for giving up privacy for an illegal activity [21]. Protecting a prefixed set of records, which is decided independent of the database, works when anomalies are defined independent of the other 
records. However, for a data-dependent anomaly definition, such a notion of privacy fails to protect the normal records. Here the problem arises due to the fixed nature of the set that is databasespecific. In the case of a data-dependent definition of anomaly, if we wish to provide privacy guarantee to the normal - call them sensitive - records that are present in the database, then specifying the set of sensitive records itself leaks information and can lead to a privacy breach. Thus, sensitive records must be defined based on a more fundamental premise to reduces such dependencies. This notion of sensitive record plays a pivotal role in defining a notion of privacy, named sensitive privacy, which is appropriate for the problem identifying anomaly.

We remark that although anomaly identification method provide binary labeling, they assign scores to represent how outlying a record is [1,3]; thus these models (implicitly or explicitly) assign a records a degree of outlyingness with respect to the other records, which the following discussion takes into account.

An appropriate notion of privacy in our setting must allow a privacy mechanism to have the following two important properties. First, the more outlying (or non-outlying) a record is, the higher the accuracy the privacy mechanism can achieve for anomaly identification, which is in contrast to DP (Figure 2c). Second, all the sensitive records should have DP like privacy guarantee for the same value of privacy parameter.

The mechanisms that are private under sensitive privacy achieve both the properties (see Figure 2, which gives the indicative experimental results on the example data; see Section A.1 for the details on the experiment and the values of the parameters). Furthermore, it has an additional property: in a typical setting, the anomalies do not lose privacy altogether; instead the more outlying a record is the lesser privacy it has (Figure 2d).

\subsection{How do we define the new privacy notion?}

To define privacy, we need a metric space over the databases since a private mechanism needs to statistically blur the distinction between databases that are close in the metric space. While differential privacy uses the $\|\cdot\|_{1}-$ metric, we utilize a different metric over databases, which can be defined using the notion of sensitive record. Informally, we say a record is sensitive with respect to a database if it is normal or becomes normal under a small change-we formalize this in Section 3. We argue that this notion of sensitive record is quite natural, and it is inspired from the existing anomaly detection literature $[1,3]$. Since, by definition, an anomalous record significantly diverges from other records in the database [1, 3], a small change in the database should not affect the label of an anomalous record. Given the definition of sensitive record, a graph over the databases is defined by adding an edge between two databases if and only if they differ in a sensitive record. The metric over the databases is now given by the shortest path length between the databases in this graph. This metric space has the property that databases differing by a sensitive record are closer compared to the databases differing in a non-sensitive record. We use the proposed metric space to define sensitive privacy, which enables us to fine-tune the tradeoff between accuracy and privacy.
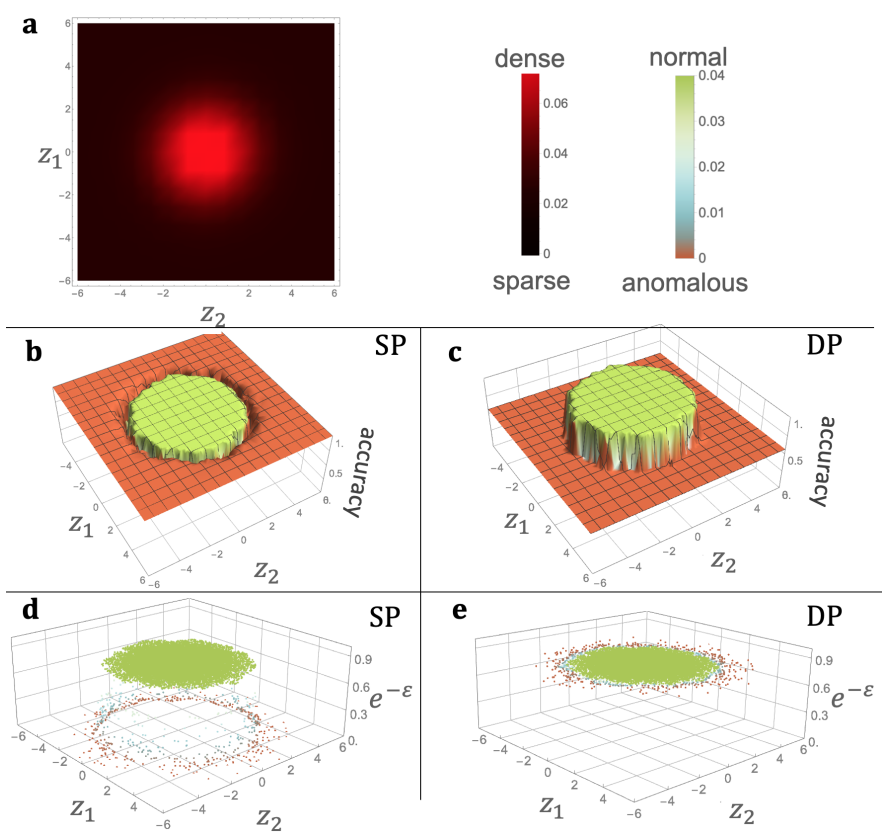

Figure 2: (b), (c) is for the same data, and (d), (e) is for the same data. (a) gives the density plot of the distribution of the example data. $z_{1}$ and $z_{2}$ axes give the coordinate of a point (record). (b) and (c) resp. show the accuracy (on vertical axis) for anomaly identification (AId) via sensitively private (SP) and DP mechanisms for the data. The plots give the interpolated results to clarify the relationship of outlyingness and accuracy. (d) and (e) give the privacy (on vertical axis) for each record in the data for private AId. All the green (normal) points in (d) are at the same level as all the points in (e).

\section{PRELIMINARIES AND NOTATION}

Database: We consider a database as a multiset of elements from a set $\mathcal{X}$, which is the set of possible values of records. In a database, we assume each record is associated with a distinct individual. We represent a database $x$ as a histogram in $\mathcal{D}=\left\{y \in \mathbb{N}^{X}:\|y\|_{1}<\infty\right\}$, where $\mathcal{D}$ is the set of all possible database, $\mathbb{N}=\{0,1,2, \ldots\}$, and $x_{i}$ is the number of records in $x$ that are identical to $i$.

Definition 2.1 (differential privacy $[15,17]$ ). For $\varepsilon>0$, a mechanism $M$ with domain $\mathcal{D}$ is $\varepsilon$-differentially private if for every $x, y \in \mathcal{D}$ such that $\|x-y\|_{1} \leq 1$, and every $R \subseteq \operatorname{Range}(M)$,

$$
P(M(x) \in R) \leq e^{\varepsilon} P(M(y) \in R) .
$$

We implicitly assume that the $R$ 's are chosen such that the events “ $M(x) \in R$ ” are measurable.

Anomalies: For any database $x$, record $i \in \mathcal{X}, r \geq 0$, and a distance function $d: \mathcal{X} \times \mathcal{X} \rightarrow \mathbb{R}_{\geq 0}, B_{\mathcal{X}}(i, r)=\sum_{j \in \mathcal{X}: d(i, j) \leq r} x_{j}$, and define $(\beta, r)$-anomaly as follows.

Definition $2.2((\beta, r)$-anomaly [35]). For a given database $x$ and record $i$, we say $i$ is a $(\beta, r)$-anomaly in the database $x$ if $i$ is present in $x$, i.e. $x_{i}>0$, and there are at most $\beta$ records in $x$ that are within distance $r$ from $i$, i.e. $B_{x}(i, r) \leq \beta$. 


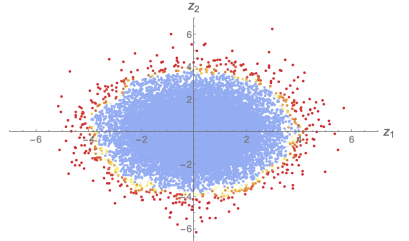

(a)

(c)

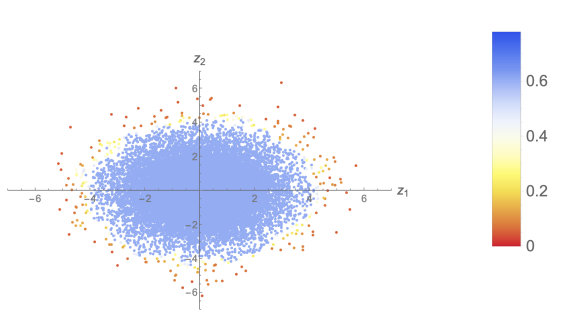

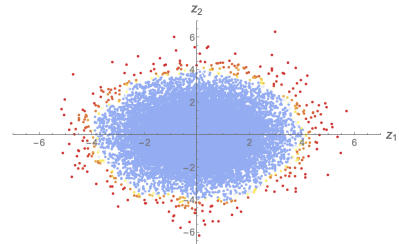

(b)
Figure 3: (a)-(c), the plot is for the same data. The two axes give the coordinate of a point (record). The color gives the level of privacy, i.e. the value $e^{-\varepsilon}$, for 0.25 -SP AIQ for every record (the data was generated using generated using the distribution given in Figure 2). (a), $k=1$. (b), $k=7$. (c), $k=14$.

Whenever we refer to a $(\beta, r)$-anomaly, we assume there is an arbitrary distance function $d$ over $\mathcal{X} \times \mathcal{X}$.

Anomaly identification: Let us now introduce the important and related notion of anomaly identification function, $g: X \times \mathcal{D} \rightarrow$ $\{0,1\}$, such that for a given anomaly definition, every record $i \in$ $\mathcal{X}$ and database $x \in \mathcal{D}, g(i, x)=1$ if and only if $i$ is present in $x$ as an anomalous record (note that no change is made to $x$ ). This formulation is extensible to the case where the database over which anomaly identification is performed is considered to include the record for which anomaly identification is desired. Here, the anomaly identification for a record $i$ over a data $x$ can be computed over the database that consists of all the records in $x$ as well as the record $i^{1}$.

Private anomaly identification query (AIQ): Here, all the private mechanisms we consider have domain $\mathcal{D}$. Thus, we will consider the anomaly identification query to be for a fixed record. We will specify this by the pair $(i, g)$, where $i$ is a record and $g$ an anomaly identification function. Now a private anomaly identification mechanism, $M: \mathcal{D} \rightarrow\{0,1\}$, for a fixed AIQ, $(i, g)$, can be represented by its distribution, where for every $x, \mathrm{P}(M(x)=g(i, x))$ is the probability the $M$ output correctly, and $\mathrm{P}(M(x) \neq g(i, x))$ is the probability that $M$ errs on $x$.

\section{SENSITIVE PRIVACY}

Our notion of sensitive privacy requires privacy protection of every record that may be normal under a small change in the database.

\footnotetext{
${ }^{1}$ Note that alternatively one could have defined $g$ without predicating on the existence of $i$ in $x$. By dropping the predicate on the existence of $i$, we in effect blur the distinction between the notion of a void spot (that in a different database could have been occupied by a record) in the database and the notion of an anomaly.
}

We use the notion of normality property $p$ to identify the normal records that exist in the database. Formally, for a given definition of anomaly, a normality property, $p: \mathcal{X} \times \mathcal{D} \rightarrow\{0,1\}$, is such that for every record $i$ and database $x, p(i, x)=1$ if and only if $i$ is present in $x$ as a normal record. Note that the normality property is not the negation of anomaly identification function because for the absent records $p=0$ (same as those which do not satisfy the property). We formalize the notion of small change in the database as the addition or removal of $k$ records from the database. We consider this change to be typical and want to protect the privacy of every record that may become normal under this small change in the database.

We now formalize the key notion of sensitive record. For a fixed normality property, all the records whose privacy must be protected are termed as sensitive records.

Definition 3.1 (sensitive record). For $k \geq 1$ and a normality property $p$, we say a record $i$ is $k$-sensitive with respect to a database $x$ if, for a database $y,\|x-y\|_{1} \leq k$ and $p(i, y)=1$.

Next, we give a couple of definitions of the graphs we consider here. A neighborhood graph, $\mathbb{G}=(\mathcal{D}, E)$, is a simple graph such that for every $x$ and $y$ in $\mathcal{D},(x, y) \in E \Longleftrightarrow\|x-y\|_{1}=1$. One of the important notions in this work is $k$-sensitive neighborhood graph, $G_{S}=\left(\mathcal{D}, E^{\prime}\right)$, for $k \geq 1$ and a normality property, which is a subgraph of the neighborhood graph, $\mathbb{G}=(\mathcal{D}, E)$, such that for every $(x, y) \in E,(x, y) \in E^{\prime} \Longleftrightarrow$ for some $i \in \mathcal{X}, \mid x_{i}-$ $y_{i} \mid=1$ and $i$ is $k$-sensitive with respect to $x$ or $y$. Further, the two databases connected by an edge in a (sensitive) neighborhood graph are called neighbors. With this, we can state the notion of sensitive privacy. Note that the $k$-sensitive neighborhood graph is tied to the normality property, and hence, the anomaly definition.

Definition 3.2 (sensitive privacy). For $\varepsilon>0, k \geq 1$, and normality property, a mechanism $M$ with domain $\mathcal{D}$ is $(\varepsilon, k)$-sensitively private if for every two neighboring databases $x$ and $y$ in $k$-sensitive neighborhood graph, and every $R \subseteq \operatorname{Range}(M)$,

$$
P(M(x) \in R) \leq e^{\varepsilon} P(M(y) \in R)
$$

We omit $k$ when it is clear from the context. The above condition necessitates that for every two neighbors, any test (i.e., event) one may be concerned about, should occur with "almost the same probability", that is, the presence or the absence of a sensitive record should not affect the likelihood of occurrence of any event. Here, "almost the same probability" means that the above probabilities are within a multiplicative factor $e^{\varepsilon}$. The guarantees provided by sensitive privacy are similar to that of differential privacy. Sensitive privacy guarantees that given the output of the private mechanism, an adversary cannot infer the presence or the absence of a sensitive record. Thus for neighboring databases in a sensitive neighborhood graph $\left(G_{S}\right)$, the guarantee is exactly the same as in differential privacy. If $x$ and $y$ differ by one record, which is not sensitive, then they are not neighbors in $G_{S}$, and the guarantee provided by sensitive privacy is weaker ${ }^{2}$ than differential privacy, nevertheless, has the same form. So, intuitively, if we only consider the databases, where all the records are sensitive, then differential privacy and sensitive privacy provide exactly the same

\footnotetext{
2"Weaker" means that every mechanism which is $\varepsilon$-DP is also $\varepsilon$-SP, but in general not the other way around.
} 
guarantee. In general, every $(\varepsilon, k)$-SP mechanism $M$ for $G_{S}$ satisfies $P(M(x) \in R) \leq P(M(y) \in R) e^{\varepsilon d_{G_{S}}(x, y)}$ for every $x, y$ and $R$, where $d_{G_{S}}$ is the shortest path length metric over $G_{S}$.

Similar to differential privacy, $\varepsilon$ is the privacy parameter: the lower its value, the higher the privacy guarantee. The parameter $k$ ,which is associated with the sensitive neighborhood graph, provides a way to quantify what is deemed as a small change in the database, which varies from field to field, but nevertheless in many common cases can be quantified over an appropriate metric space. ${ }^{3}$. When we increase the value of $k$, we move the boundary between what is considered sensitive and what is non-sensitive (Figure 3, where the plots are similar to the ones given in Figure $2 \mathrm{~d}$ for the same parameter values but for varying $k$ ): higher the value of $k$, the more records are considered sensitive, and therefore, must be protected. This is due to the fact that, for any $k \geq 1$, if a record is $k$-sensitive with respect to a database $x$, it is also $(k+1)$-sensitive with respect to $x$. For example, with respect to a database $x$, a 2sensitive record, may not be 1-sensitive, but a 1-sensitive record will also be 2-sensitive.

\subsection{Composition}

Our formalization of sensitive privacy enjoys the important properties of composition and post-processing [18], which a good privacy definition should have [32]. Hence, we can quantify how much privacy may be lost (in terms of the value of $\varepsilon$ ) if one asks multiple queries or post-processes the result of a private mechanism. Here, we recall that sensitive privacy is defined with respect to the $k$-sensitive neighborhood graph for the privacy parameter $\varepsilon$. Thus, the privacy composes with respect to both, the privacy parameter (i.e. $\varepsilon$ ) and the sensitive neighborhood graph.

Sequential composition provides the privacy guarantee over multiple queries over the same database, where the same record(s) in the database may be used to answer more than one query. Consider two mechanisms $M_{1}: \mathcal{D} \rightarrow R$, which is $\varepsilon_{1}$-sensitively private for $k_{1}$ sensitive neighborhood graph $G_{S_{1}}=\left(\mathcal{D}, E_{1}\right)$, and $M_{2}: \mathcal{D} \times R \rightarrow R^{\prime}$, which is $\varepsilon_{2}$-sensitively private for $k_{2}$-sensitive neighborhood graph $G_{S_{2}}=\left(\mathcal{D}, E_{2}\right)$, with independent sources of randomness. Recall that for a private mechanisms for AIQ, $(i, g)$, is fixed; thus $M_{1}$ and $M_{2}$ may correspond to different records and anomaly identification function. Now, $M_{2}\left(x, M_{1}(x)\right)$ (for every database $\left.x\right)$ is $\left(\varepsilon_{1}+\varepsilon_{2}\right)$ sensitively private for $G_{S}=\left(\mathcal{D}, E_{1} \cap E_{2}\right)$ (Claim 3$)$. One application of this is that for a fixed $G_{S}$, even performing multiple queries interactively will lead to at most a linear loss (in terms of $\varepsilon$ ) in privacy in the number of queries-in an interactive query over a database $x$, one firstly gets the answer of $M_{1}$, i.e., $M_{1}(x)$, and based on the answer, one selects $M_{2}$ and gets its answer. Furthermore, for a fixed normality property, if $k_{1} \leq k_{2}$ then $G_{S_{1}}$ is a subgraph of $G_{S_{2}}$, then $M_{2}$ is $\left(\varepsilon_{1}+\varepsilon_{2}\right)$-sensitively private for $G_{S_{1}}$.

Parallel composition deals with multiple queries, each of which only uses non-overlapping partition of the database. Let $X=Y_{1} \cup Y_{2}$ such that $Y_{1} \cap Y_{2}=\emptyset$. Now, consider $M_{1}$ and $M_{2}$, each with domain $\mathcal{D}$, that are respectively $\varepsilon_{1}$-sensitively private for $G_{S_{1}}$ and $\varepsilon_{2}$ sensitively private for $G_{S_{2}}$, where $G_{S_{1}}$ is a subgraph of $G_{S_{2}}$. Further,

\footnotetext{
${ }^{3}$ The metric space we are using for anomaly identification has a rather complicated structure, but it is induced by formalizing our intuition for sensitive records.
}

$M_{1}$ and $M_{2}$ only depend on their randomness (each with its independent source) and records in $Y_{1}$ and $Y_{2}$ respectively. In this setting, a mechanism $M(x)=\left(M_{1}(x), M_{2}(x)\right)$ is $\max \left(\varepsilon_{1}, \varepsilon_{2}\right)$-sensitively private for $G_{S_{1}}$, or in general case for sensitive neighborhood graph $\left(\mathcal{D}, E_{1} \cap E_{2}\right)$ (Claim 4), where $E_{1}$ and $E_{2}$ are the sets of edges for $G_{S_{1}}$ and $G_{S_{2}}$ respectively.

We also remark that privacy is maintained under post-processing.

Example: Consider composition for sensitive privacy for the case of multiple $(\beta, r)$-AIQs. Let us say we answer anomaly identification queries for records $i_{1}, i_{2}, \ldots, i_{n}$ respectively for $\left(\beta_{1}, r_{1}\right)$, $\left(\beta_{2}, r_{2}\right), \ldots,\left(\beta_{n}, r_{n}\right)$ anomalies over the database $x$, while providing sensitive privacy. Let the mechanism for answering $\left(\beta_{t}, r_{t}\right)$-AIQ for $i_{t}$ be $\varepsilon_{t}$-SP for $k_{t}$-sensitive neighborhood graph corresponding to $\left(\beta_{t}, r_{t}\right)$-anomaly, and assume it depends on the partition of the database that contains the records within distance $r_{t}$ of $i_{t}$ (because it suffices to compute $\left(\beta_{t}, r_{t}\right)$-AIQ) and its independent source of randomness. Let $k=\min \left(k_{1}, \ldots, k_{n}\right), \beta=\max \left(\beta_{1}, \ldots, \beta_{n}\right)$, and $r=\min \left(r_{1}, \ldots, r_{n}\right)$. In this case, the sensitive privacy guarantee for answering all of the queries is $m \varepsilon$ for $k$-sensitive neighborhood graph corresponding to $(\beta, r)$-anomaly, where $m$ is the maximum number of $i_{t}$ 's that are within any ball of radius $\max \left(r_{1}, \ldots, r_{n}\right)$ (Claim 5).

Thus, from the above, it follows that if we fix $\beta, r$ and $k$ and allow a querier to ask $m$ many $\left(\beta^{\prime}, r\right)$-AIQ's (each may have a different value for $\beta^{\prime}$ ) such that $\beta^{\prime} \leq \beta$, then we can answer all of the queries with sensitive privacy $m \varepsilon$ in the worst case for $k$-sensitive neighbor for to $(\beta, r)$-anomaly. The same is true if the queries are for $\left(\beta, r^{\prime}\right)$ with $r^{\prime} \geq r$. Furthermore, for fixed $\beta, r$ and $k$, answering $(\beta, r)$-AIQ for $i$ and $i^{\prime}$ such that $d\left(i, i^{\prime}\right)>2 r$ still maintains $(\varepsilon, k)$-SP. One may employ this to query adaptively to carry out the analysis while providing sensitive privacy guarantees over analysis as a whole.

\section{PRIVACY MECHANISM CONSTRUCTIONS}

In this section we will show how to construct a private mechanisms for $(\beta, r)$-anomaly identification. Specifically, (i) we will give an SP mechanism that errs with exponentially small probability on most of the typical inputs (Theorem 4.6), (ii) we will provide a DP mechanism construction for $(\beta, r)$-AIQ, which we will prove is optimal (Theorem 4.4), (iii) we will present a compiler construction that can compile a "bad" DP mechanism for AIQ to a "good" SP mechanism (Theorem 4.7) - here good and bad are indicative of utility. We will use these mechanism to evaluate the performance of our method over real world and synthetic datasets.

Recall that a privacy mechanism, $M: \mathcal{D} \rightarrow\{0,1\}$, for a fixed AIQ, $(i, g)$, will output the labels of $i$ for the given database, where $g$ is an anomaly identification function and $i$ is a record. The sensitive privacy requires that the shorter the distance between any two databases, $x$ and $y$, in the sensitive neighborhood graph $\left(G_{S}\right)$, the closer the probabilities of any output $(R)$ of the mechanism $M$ corresponding to the two databases should be, that is, $e^{-\varepsilon d_{G_{S}}(x, y)} \leq \mathrm{P}(M(x)=R) / \mathrm{P}(M(y)=R) \leq e^{\varepsilon d_{G_{S}}(x, y)}$. Thus, for an $x$, the greater is the distance to the closest $y$ such that $g(i, x) \neq g(i, y)$, the higher accuracy a private mechanism can achieve on the input $x$ for answering $g(i, x)$. We capture this metricbased property by the minimum discrepant distance (mdd) function. 




Figure 4: Sensitive neighborhood graph. A simple example of a 1-sensitive neighborhood graph, $G_{S}$, with $X=\{1,2,3,4,5\}, \ell_{1}$ metric over $\mathcal{X} \times \mathcal{X}$, and $(\beta=3, r=1)$-anomaly. Note that $G_{S}$ is an undirected graph; arrowheads indicates the record is added at the end node; the color of the edge corresponds (as per the given color code) to the value of the record added. Further, each database $x$ is represented as a 5-tuple with $x_{i}$ for $i \in \mathcal{X}$ representing the number of records in $x$ that have value $i$.

Fix an anomaly identification function $g$. For a given sensitive neighborhood graph $G_{S}, \Delta_{G_{S}}$ is $m d d$-function, if for every $i$ and $x$,

$$
\Delta_{G_{S}}(i, x)=\min _{y \in \mathcal{D}: g(i, y) \neq g(i, x)} d_{G_{S}}(x, y)
$$

A simple and efficient mechanism for anomaly identification that is both accurate and sensitively private can be given if $g$ and the $\Delta_{G_{S}}$ (the corresponding mdd-function) can be computed efficiently. However, computing the mdd-function efficiently for an arbitrary anomaly definition is a non-trivial task. This is because the metric, $d_{G_{S}}$, which gives rise to the metric-based property captured by the mdd-function, is induced by (a) the definition of anomaly (e.g. specific values of $\beta$ and $r$ ) and (b) the metric over the records. Thus, making it exceedingly difficult to analyze it in general.

We use the example given in Figure 4 to explain the above mentioned relationships of mdd-function. This figure depicts a subgraph of 1-sensitive neighborhood graph for $(\beta=3, r=1)$ anomaly. One can appreciate the conceptual difficulty in calculating mdd-function, $\Delta_{G_{S}}$ (for this setting) by for example thinking the value of $\Delta_{G_{S}}(5,(3,0,0,0,1))$ (and recall that this is just a 1-sensitive neighborhood graph). Next, note that for a given database $x$ and a record $i$, the shorter is the distance of the closest sensitive record from $i$, the smaller the value of $\Delta_{G_{S}}(i, x)$, e.g. $\Delta_{G_{S}}(5,(3,0,0,0,1))>\Delta_{G_{S}}(5,(3,2,1,0,1))$. Furthermore, the presence of non-sensitive records can also influence the value of the mdd-function, e.g. $\Delta_{G_{S}}(5,(3,0,0,0,1))>\Delta_{G_{S}}(5,(3,0,1,0,1))$ although the closest sensitive record to 5 is the same in both the databases. In addition, the values of $\beta$ and $r$ also affect the value of mdd-function, and in most realistic settings, the size of $\mathcal{X}$ is large, and the sensitive neighborhood graph is quite complex.

Below, we provide our constructions that uses a lower bound on the mdd-function to give sensitively private mechanism, which does not depend upon any particular definition of anomaly. Thus it can be used to give private mechanisms for AIQ's as long as one is able to compute the lower bound.

\subsection{Construction: SP-mechanism for AIQ by lower bounding mdd-function}

Here, we show how to construct an SP mechanism for identifying anomalies by using a lower bound, $\lambda$, for the mdd-function. Our construction (Construction 1) will be parameterized by $\lambda$, which is associated with a sensitive neighborhood graph. Since the sensitive neighborhood graph is tied to an anomaly definition, it will become concrete once we give the definition of anomaly (e.g., see Section 4.1.1 and Section 4.1.2).

For any fixed AIQ, $(i, g)$, and given $\lambda$, Construction 1 provably gives an SP mechanism as long as $\lambda$ fulfills the following two properties: (1) for every $i$ and $x, \lambda(i, x) \geq 1$ and (2) $\lambda$ is 1-Lipschitz continuous lower bound on the mdd-function (Theorem 4.1).

For a sensitive neighborhood graph, $G_{S}$, we say a function $f$ : $\mathcal{X} \times \mathcal{D} \rightarrow \mathbb{R}$ is $\alpha$-Lipschitz continuous if for every $i \in \mathcal{X}$ and neighboring databases $x$ and $y$ in $G_{S},|f(i, x)-f(i, y)| \leq \alpha$.

We remark that although at first it appears that the Lipschitz continuity condition is some side technicality, in fact bounding its value constitute the main part of our argument for privacy of our 
mechanisms. Thus giving an SP mechanism for $(i, g)$ via Construction 1 reduces to giving a Lipschitz continuous lower bound for the mdd-function corresponding to $g$.

Construction 1. $U_{\lambda}$

(1) Input $x \in \mathcal{D}$.

(2) Set $t=e^{-\varepsilon(\lambda(i, x)-1)} /\left(1+e^{\varepsilon}\right)$.

(3) Sample $b$ from $\{0,1\}$ such that $\mathrm{P}(b \neq g(i, x))=t$.

(4) Return b.

Note that the above is a family of constructions parameterized by $\lambda$ (as mentioned above), i.e., one construction, $U_{\lambda}$, for each $\lambda$. This construction is very efficiently realizable as long as we can efficiently compute $g$ and $\lambda$. Furthermore, the error of the mechanism, yielded by the construction, for any input is exponentially small in $\lambda$ (Claim 2, which immediately follows from the construction).

Claim 2. For given $\varepsilon,(i, g)$, and $\lambda, U_{\lambda}$ (Construction 1) is such that $P(U(x) \neq g(i, x))=e^{-\varepsilon(\lambda(i, x)-1)} /\left(1+e^{\varepsilon}\right)$ for every $x$.

Theorem 4.1 ( $U_{\lambda}$ IS SP). For any given $\varepsilon$, AIQ, and a 1-Lipschitz continuous lower bound $\lambda$ on the corresponding mdd-function for $k$-sensitive neighborhood graph, $G_{S}$, such that $\lambda \geq 1$, Construction 1 yields an $(\varepsilon, k)$-sensitively private mechanism.

In order to show that the theorem holds, it suffices to verify that for every $i$ and every two neighboring $x$ and $y$ in $G_{S}$, the privacy constraints hold. For any AIQ, $(i, g)$, this is immediate when $g(i, x)=g(i, y)$ because $\lambda$ is 1-Lipschitz continuous. When $g(i, x) \neq$ $g(i, y), \lambda(i, x)=\lambda(i, y)=1$ because $\Delta_{G_{S}}(i, x)=\Delta_{G_{S}}(i, y)=1$ and $\lambda \geq 1$. Thus, the constraints are satisfied in this case as well. The complete proof for Theorem 4.1 is given in Appendix A.4. Additionally, a simple observation on the proof of Theorem 4.1, shows that if the given $\lambda$ is $\alpha$-Lipschitz continuous with $\alpha \geq 1$, then Construction 1 yields an $(\varepsilon \cdot \alpha)$-sensitively private mechanism.

In the following two sections, we instantiate Construction 1 to give differentially private and sensitively private mechanisms for performing $(\beta, r)$-anomaly identification query. We will use these mechanisms in our empirical evaluation over real world datasets.

4.1.1 Optimal DP-mechanism for $(\beta, r)$-AIQ. Here, we show how to use Construction 1 to give an optimal differentially private mechanism for $(\beta, r)$-AIQ. Note that we will use this mechanism in experimental evaluation (Section 5) and compare its performance with our SP mechanism (which we will present shortly). We begin by restating the definition of DP in terms of the neighborhood graph. This restatement will immediately establish that SP generalizes DP, a fact we will use to build DP mechanism.

Definition 4.2 (DP restated with neighborhood graph). For $\varepsilon>0$, a mechanism, $M$, with domain $\mathcal{D}$, is $\varepsilon$-differentially private if for every two neighboring databases, $x$ and $y$, in the neighborhood graph, and every $R \subseteq \operatorname{Range}(M)$,

$$
P(M(x) \in R) \leq e^{\varepsilon} P(M(y) \in R) .
$$

From Definition 3.2 (of sensitive privacy) and Definition 4.2, it is clear that differential privacy is a special case of sensitive privacy, when the $k$-sensitive neighborhood graphs, $G_{S}$, is the same as neighborhood graph, $\mathbb{G}$, i.e., $G_{S}=\mathbb{G}$. Thus, for $G_{S}=\mathbb{G}$, a mechanism is $\varepsilon$-differentially private if and only if it is $\varepsilon$-sensitively private. This observation is sufficient to give a differentially private mechanism for AIQ by using Construction 1.

We use $\lambda=\Delta_{\mathbb{G}}$ in Construction 1 to give the DP mechanism for $(\beta, r)$-AIQ, where $\Delta_{\mathbb{G}}$ (mdd-function) for an arbitrary $\beta, r, i$ and $x$ is given below. This will yield a DP mechanism as long as the given $\Delta_{\mathbb{G}}$ for $(\beta, r)$-AIQ is 1-Lipschitz continuous, a fact that immediately follows from the above observation and Theorem 4.1. We claim that for any given $\beta$ and $r, \Delta_{\mathbb{G}}$ (given by (2)) is mdd-function for the $(\beta, r)$-AIQ and is 1-Lipschitz continuous (Lemma 4.3).

$$
\Delta_{\mathbb{G}}(i, x)= \begin{cases}1 & x_{i}=0 \wedge B_{x}(i, r)<\beta \\ 2+B_{x}(i, r)-\beta & x_{i}=0 \wedge B_{x}(i, r) \geq \beta \\ \min \left(x_{i}, \beta+1-B_{x}(i, r)\right) & x_{i}>0 \wedge B_{x}(i, r) \leq \beta \\ B_{x}(i, r)-\beta & x_{i}>0 \wedge B_{x}(i, r)>\beta\end{cases}
$$

Lemma 4.3. For any fixed $(\beta, r)-A I Q,(i, g)$, the $\Delta_{\mathbb{G}}$ given by $(2)$ is mdd-function for $g$ and is 1-Lipschitz continuous.

The proof of Lemma 4.3 can be found in Appendix A.5.

We claim that for any fixed $(\beta, r)$-AIQ, $(i, g), U_{\Delta_{G}}$ (given by our construction) is differentially private and errs minimum for all the inputs (Theorem 4.4), namely, it is pareto optimal. We say $U_{\Delta_{\mathbb{G}}}$ is pareto optimal $\varepsilon$-DP mechanism if (a) it is $\varepsilon$-DP and (b) for every $\varepsilon$-DP mechanism $M: \mathcal{D} \rightarrow\{0,1\}$ and every database $x \in \mathcal{D}$, $P\left(U_{\Delta_{\mathbb{G}}}(x)=g(i, x)\right) \geq P(M(x)=g(i, x))$. Particularly, this implies that of all the DP mechanisms yielded by Construction 1 , each corresponding to a different $\lambda$, the "best" mechanism is for $\lambda=\Delta_{\mathbb{G}}$.

THeOREM $4.4\left(U_{\Delta_{G}}\right.$ IS OPTIMAL AND DP). For any fixed $(\beta, r)$ $A I Q, U_{\Delta_{G}}$ (Construction 1) is pareto optimal $\varepsilon$-differentially private mechanism, where $\Delta_{\mathbb{G}}$ is given by (2).

4.1.2 SP-mechanism for $(\beta, r)$-AIQ. We employ Construction 1 to give a $(\varepsilon, k)$-sensitively private mechanism for $(\beta, r)$-AIQ. We provide $\lambda_{k}$ below, which is 1-Lipschitz continuous lower bound on the mdd-function for the $k$-sensitive neighborhood graph for $(\beta, r)$-anomaly (Lemma 4.5). For the $\lambda_{k}$, Construction 1 yields $U_{\lambda_{k}}$ that is $(\varepsilon, k)$-SP mechanism, and for non-sensitive records $U_{\lambda_{k}}$ can have exponentially small error in $\beta$ (Theorem 4.6).

$$
\lambda_{k}(i, x)= \begin{cases}\Delta_{\mathbb{G}}(i, x) & B_{x}(i, r) \geq \beta+1-k \\ \beta+1-B_{x}(i, r) & \\ +\min \left(0, x_{i}-k\right) & B_{x}(i, r)<\beta+1-k\end{cases}
$$

Lemma 4.5. Arbitrarily fix $k, \beta \geq 1$ and $r \geq 0$. Let $g$ be $(\beta, r)$ anomaly identification function and $\Delta_{G_{S}}$ be the mdd-function for $g$, where $G_{S}$ is the $k$-sensitive neighborhood graph for $(\beta, r)$-anomaly. The $\lambda_{k}$ given by (3) is 1-Lipschitz continuous lower bound on $\Delta_{G_{S}}$.

The proof of Lemma 4.5 is given in Appendix A.7.

It is clear form the definition of $\lambda_{k}$ (given by 3 ) that when a record, $i$, is $k$-sensitive with respect to $x, \lambda_{k}(i, x)=\Delta_{\mathbb{G}}(i, x)$, which implies that there is no gain in utility (i.e. accuracy) compared to the optimal DP mechanism (in Section 4.1.1). However, when a record is not sensitive, $\lambda(i, x)>\Delta_{\mathbb{G}}(i, x)$, our SP mechanism achieves much higher utility compared to the optimal DP mechanism, which is especially true for strong $(\beta, r)$-anomalies (i.e. the records that lie in a very sparse region). 
THEOREM 4.6 (ACCURACY AND PRIVACY OF $U_{\lambda_{k}}$ ). Fix any $(\beta, r)$ AIQ, $(i, g)$. The mechanism, $U_{\lambda_{k}}$ (Construction 1 for $\lambda_{k}$ above) is $(\varepsilon, k)$-SP such that for every $i$ and $x$, if $i$ not sensitive for $x$, then $P\left(U_{\lambda_{k}}(x) \neq g(i, x)\right) \leq e^{-\varepsilon\left|\beta+1-k-B_{x}(i, r)\right|}$.

The privacy claim follows from Lemma 4.5 and Theorem 4.1, while the accuracy claim is an immediate implication from Construction 1 based on the definitions of $\Delta_{\mathbb{G}}$ and $\lambda_{k}$ - note that $B_{x}(i, r)<\beta+1-k$ implies $i$ is not sensitive for $x$ (Lemma A.2).

We give an example to show that $U_{\lambda_{k}}$ achieves high accuracy in typical settings. Fix $k \leq \beta / 10$. Now for any record $i$ in a database $x$, satisfying $B_{x}(i, r) \leq \beta / 2$ is an outlier for which $U_{\lambda_{k}}$ will err with probability less that $e^{-2 \varepsilon \beta / 5}$.

\subsection{Compiler for SP-mechanism for AIQ}

In this section, we present a construction compiler, which compiles a differentially private mechanism for an anomaly identification query into a sensitively private one. This SP mechanism can outperform the differentially private mechanism. Furthermore, our compiler is not specific to any particular definition of anomaly or any specific DP mechanism. The differentially private mechanism, which the compiler takes, is given in terms of its distribution over the outputs for every input. The compiled SP mechanism comparatively has much better accuracy for the non-sensitive records; however, for the sensitive records, the SP and the input DP mechanism err by the same amount.

It is noteworthy that for many problems, we already know the distributions given by differentially private mechanisms $[15,17,18]$. Thus, our construction can be employed using these mechanism as long as the distributions given by the differentially private mechanism are not too "wild", for example, the probability of the wrong answer for any input is not too high (we formalize this below), which is typically true.

The compiler construction is parameterized by $\delta$. This $\delta$ must be a non-negative lower bound on $\Delta_{G_{S}}-\Delta_{\mathbb{G}}$ that is also 2-Lipschitz continuous $\left(\Delta_{G_{S}}\right.$ and $\Delta_{\mathbb{G}}$ are the mdd-functions for an arbitrarily fixed $g$, and $G_{S}$ is the $k$-sensitive neighborhood graph for anomaly definition for $g$ ). The non-negativity constraint is a side technicality; however, bounded divergence (i.e., the Lipschitz continuity constraint) and the lower bound constraint play a pivotal role in arguing bout the privacy of the compiled mechanism. Given below is our construction, and it will be useful when obtaining $\delta$ is easier than $\lambda$, and we already know the distributions of a DP mechanism for the problem.

\section{Construction 2. $U_{\delta}$}

(1) Input $x \in \mathcal{D}$.

(2) Set $t=\mathrm{P}(M(x) \neq g(i, x)) / e^{\frac{\varepsilon}{4} \delta(i, x)}$.

(3) Sample $b$ from $\{0,1\}$ such that $\mathrm{P}(b \neq g(i, x))=t$.

(4) Return $b$.

The differentially private mechanism (in terms of its distributions) that can be transformed (with provable guarantees) through our compiler is termed as a valid mechanism. For $\varepsilon>0$ and any fixed AIQ, $(i, g)$, we say an $\varepsilon$-DP mechanism, $M: \mathcal{D} \rightarrow\{0,1\}$, is valid if for every two neighbors $x$ and $y$ in the neighborhood graph with $g(i, x)=g(i, y)=b$ for some $b \in\{0,1\}$, the following holds

$$
1-\mathrm{P}(M(x) \neq b) e^{-\varepsilon} \leq e^{2 \varepsilon}(1-\mathrm{P}(M(y) \neq b)) .
$$

Note that any $\varepsilon$-differentially private mechanism, $M$, for a fixed AIQ, $(i, g)$, that satisfies $\mathrm{P}(M(x) \neq g(i, x)) \leq e^{2 \varepsilon} /\left(1+e^{2 \varepsilon}\right)$ for every $x$ is valid - this is shown below for $\varepsilon>0$ and two arbitrary neighbors $x$ and $y$ such that $b=g(i, x)=g(i, y)$; hence the notion of valid differentially private mechanism is well defined.

$$
\begin{gathered}
\mathrm{P}(M(y) \neq b) \leq \frac{e^{2 \varepsilon}}{e^{2 \varepsilon}+1} \Longrightarrow \\
\mathrm{P}(M(y) \neq b) e^{4 \varepsilon}-\mathrm{P}(M(y) \neq b) \leq e^{2 \varepsilon}\left(e^{2 \varepsilon}-1\right) \\
\text { since } M \text { is } \varepsilon \text { - } \mathrm{PP}, \text { it follows from the above } \\
\mathrm{P}(M(y) \neq b) e^{4 \varepsilon}-\mathrm{P}(M(x) \neq b) e^{\varepsilon} \leq e^{2 \varepsilon}\left(e^{2 \varepsilon}-1\right) \Longrightarrow \\
1-\mathrm{P}(M(x) \neq b) e^{-\varepsilon} \leq e^{2 \varepsilon}(1-\mathrm{P}(M(y) \neq b))
\end{gathered}
$$

We claim that for a given valid differentially private mechanism, $M$, for a fixed AIQ, $(i, g)$, and non-negative 2-Lipschitz continuous lower bound $\delta$ on $\Delta_{G_{S}}-\Delta_{\mathbb{G}}$, Construction 2 complies $M$ into a sensitively private mechanism, $U_{\delta}$ (Theorem 4.7 ). We stress that for the compiled SP mechanism, the probability of error can be exponentially smaller compared to the input DP mechanism. which is especially true for the non-sensitive records. This leads to an improvement in accuracy. Clearly, as the input mechanism, $M$, to the compiler becomes better (i.e., has lower error) so does the compiled sensitively private mechanism, $U_{\delta}$, since the error of $U_{\delta}$, is never more than that of $M$.

THEOREM 4.7. For $k \geq 1$ and a given valid $\varepsilon / 2-D P$ mechanism, $M$, for any AIQ, $(i, g)$, and non-negative 2-Lipschitz continuous lower bound, $\delta$, on $\Delta_{G_{S}}-\Delta_{\mathbb{G}}$, Construction 2 yields an $\varepsilon-S P$ mechanism, $U_{\delta}$, for $k$-sensitive neighborhood graph corresponding to the anomaly definition for $g$ such that

$$
P\left(U_{\delta}(x) \neq g(i, x)\right)=P(M(x) \neq g(i, x)) e^{-\frac{\varepsilon}{4} \delta(i, x)} .
$$

To confirm the above claim, we show that the mechanism, $U_{\delta}$, given by the construction above indeed satisfies the privacy constraints imposed by the sensitive privacy definition for every two neighboring databases in $k$-sensitive neighborhood graph. We can accomplish this by showing that the privacy constraints are satisfied by any two arbitrarily picked neighbors, $x$ and $y$, for an arbitrarily picked valid $\varepsilon / 2$-differentially private mechanism, $M$, for an anomaly identification query, $(i, g)$ and a $\delta$ as specified above. We can divide the argument into two cases, and confirm in each case that the privacy constraints are satisfied. Case 1: $\delta(i, x)=\delta(i, y)=0$, which follows due to $M$ being differentially private; because if $M$ is $\varepsilon / 2$-differentially private then it is also $\varepsilon$-differentially private. Case 2: $\delta(i, x)>\delta(i, y) \geq 0$ - this is without loss of generality since $x$ and $y$ are picked arbitrarily. This case holds because of the following: $M$ is valid $\varepsilon / 2$-differentially private, $\delta$ is non-negative and 2-Lipschitz continuous, $g(i, x)=g(i, y)$ (because for neighboring $x$ and $y, \Delta_{G_{S}}(i, x)-\Delta_{\mathbb{G}}(i, x) \geq \delta(i, x)>0$ implies $\left.\Delta_{G_{S}}(i, x) \geq 2\right)$. We give the complete proof of Theorem 4.7 in Appendix A.8.

We highlight the effectiveness of the compiler by instantiating it for $\delta(i, x)=\lambda_{1}(i, x)-\Delta_{\mathbb{G}}(i, x)$ for every $i$ and $x$ for $(\beta, r)$-anomaly. Figure 5 shows the compilation of two DP mechanisms for $(\beta, r)$ AIQ, which widely differ in their performance. As expected, the compiled SP-mechanism outperforms the input DP-mechanism.

In Figure 5a, the input DP mechanism, $M$, has a constant error for every input database, that is, $1 /\left(1+e^{\varepsilon}\right)$ for fixed $\varepsilon=0.25$. Clearly, 


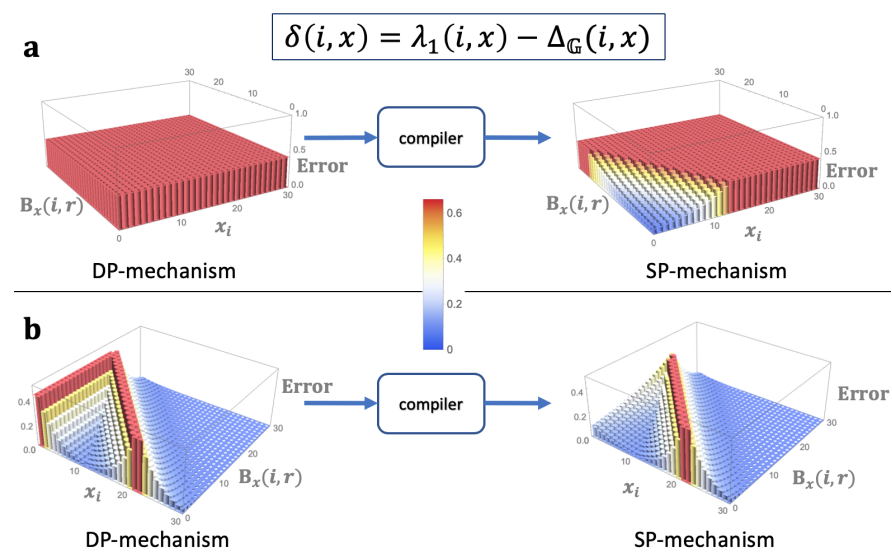

Figure 5: compilation of DP-mechanism for $(\beta, r)$-AIQ into SP-mechanism. In both (a) and (b), the input mechanism is 0.25 -DP for a fixed record $i$ and $\delta$ (given in the figure). Each database $x$ is given by $\left(x_{i}, B_{x}(i, r)\right)$ since $(\beta, r)$-anomaly identification function only depends upon $x_{i}$ and $\left.B_{x}(i, r)\right)$. Each mechanism is depicted by its error over databases i.e. $P(M(x) \neq g(i, x))$. (a), DP-mechanism has constant error $\approx$ 0.44. (b), DP-mechanism has error $\approx 0.56 / e^{0.25 \Delta_{\mathbb{G}}(i, x)}$.

this mechanism has extremely bad accuracy. This is a difficult case even for the compiled mechanism, which nevertheless, attains exponential gain in accuracy for non-sensitive records. However, when we input the DP-mechanism given in Section 4.1.1, which is much better than the one in Figure 5a, the compiled mechanism is clearly superior compared to the one in Figure $5 a$ (Figure $5 b$ ).

Note that the $\delta$ in Figure 5 is a non-negative 2-Lipschitz continuous lower bound on $\Delta_{G_{S}}-\Delta_{\mathbb{G}}$ (as required by Theorem 4.7), where $\lambda_{1}$ is given by (3) for $k=1$ and $\Delta_{\mathbb{G}}$ is given by (2). $\delta=\lambda_{1}-\Delta_{\mathbb{G}} \geq 0$ follows because $\Delta_{G_{S}} \geq \lambda_{1} \geq \Delta_{\mathbb{G}}$. The first inequality follows from Lemma 4.5. The second one trivially holds true for all the cases except for $x_{i} \geq 1$ and $B_{x}(i, r)<\beta$, where $\lambda_{1}(i, x)=\beta+$ $1-B_{x}(i, r)$ and $\Delta_{\mathbb{G}}(i, x)=\min \left(x_{i}, \beta+1-B_{x}(i, r)\right)$; thus, even in this case, we get $\delta(i, x)=\max \left(\beta+1-B_{x}(i, r)-x_{i}, 0\right) \geq 0$. The 2-Lipschitz continuity of $\delta$ follows from the $\lambda_{1}$ and $\Delta_{\mathbb{G}}$ being 1Lipschitz continuous (Lemma 4.5 and Lemma 4.3). Thus, for any $i$ and two neighbors $x$ and $y$ in $G_{S}$ (1-sensitive neighborhood graph),

$|\delta(i, x)-\delta(i, y)| \leq\left|\lambda_{1}(i, x)-\lambda_{1}(i, y)\right|+\left|\Delta_{\mathbb{G}}(i, x)-\Delta_{\mathbb{G}}(i, y)\right| \leq 2$.

Remark: We emphasize that both of our constructions are not tied to any specific definition of anomaly, and even the requirement of Lipschitz continuity is due to privacy constraints.

\section{EMPIRICAL EVALUATION}

To evaluate the performance of the SP-mechanism for $(\beta, r)$-anomaly identification, we carry out several experiments on synthetic dataset and real-world datasets from diverse domains: Credit Fraud [10] (available at Kaggle [23]), Mammography and Thyroid (available at Outlier Detection DataSets Library [41]), and APS Trucks (APS Failure at Scania Trucks, available at UCI machine learning repository [14]). Table 1 provides the datasets specifications.

To generate the synthetic data, we followed the strategy of Dong et al. [12], which is standard in the literature. The synthetic data

\begin{tabular}{|l|c|c|c|c|}
\hline Dataset & size & $\operatorname{dim}$ & $(\boldsymbol{\beta}, \boldsymbol{r})$ & $\begin{array}{c}\text { true }(\beta, \boldsymbol{r})- \\
\text { anomalies }\end{array}$ \\
\hline Credit Fraud & 284,807 & 28 & $(1022,6.7)$ & 103 \\
\hline APS Trucks & 60,000 & 170 & $(282,16.2)$ & 677 \\
\hline Synthetic & 20,000 & 200 & $(97,3.8)$ & 201 \\
\hline Mammography & 11,183 & 6 & $(55,1.7)$ & 75 \\
\hline Thyroid & 3,772 & 6 & $(18,0.1)$ & 61 \\
\hline
\end{tabular}

Table 1: dataset specifications and parameter values.

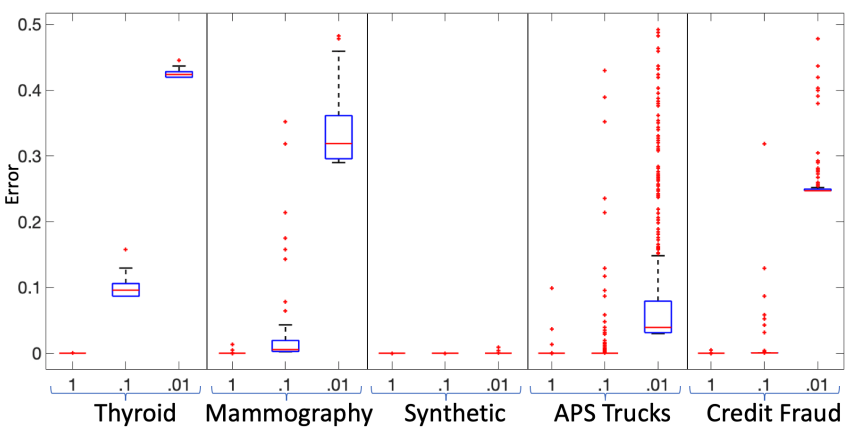

Figure 6: box plots of the errors of the SP mechanism for $(\beta, r)$-AIQ over the true $(\beta, r)$-anomalies for $\varepsilon=\{.01, .1,1\}$.

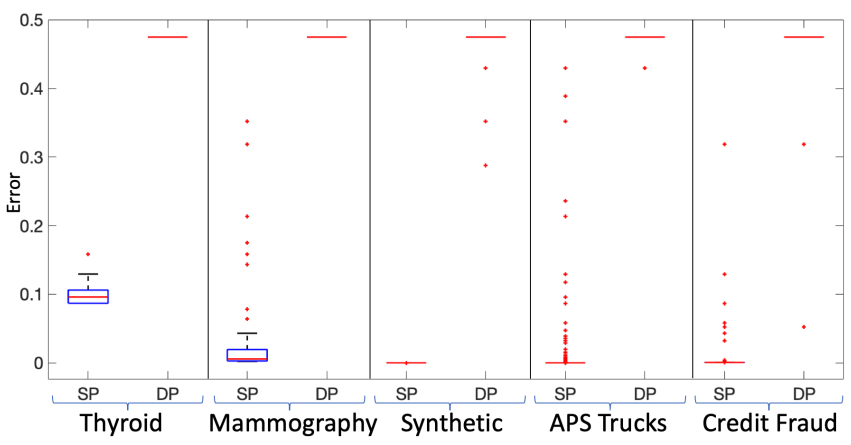

Figure 7: box plots of the error of the SP and the DP mechanisms for $(\beta, r)$-AIQ over the true $(\beta, r)$-anomalies for $\varepsilon=0.1$.

was generated from a mixed Gaussian distribution, given below, where $\mathrm{I}$ is the identity matrix of dimension $d \times d, \sigma<<1$, and $e_{i_{t}}$ is a standard base. In our experiments, we used $\rho=.01$ and $a=5$, and chose $a$ standard bases uniformly at random.

$(1-\rho) \mathcal{N}(\mathbf{0}, \mathbf{I})+\sum_{t=1}^{a}(\rho / a)\left[\frac{1}{2} \mathcal{N}\left(\sqrt{d / \rho} \mathbf{e}_{i_{t}}, \sigma^{2} \mathbf{I}\right)+\frac{1}{2} \mathcal{N}\left(-\sqrt{d / \rho} \mathbf{e}_{i_{t}}, \sigma^{2} \mathbf{I}\right)\right]$

The aim of this work is to study the effect of privacy in identifying anomalies. So we keep the focus on evaluating the proposed approach for achieving privacy for this problem, and how it compares to differential privacy in real world settings. Our experiments make use of (popular) $(\beta, r)$ notion of anomaly.

Following the standard practice for identifying outliers in the data with higher dimension [1,28], we carried out the principal component analysis (PCA) to reduce the dimension of the three datasets with higher dimension. We chose, top 6, 9, and 12 features for the Credit Fraud, Synthetic, and APS Trucks datasets respectively. Next, we obtain the values of $\beta$ and $r$, which typically 


\begin{tabular}{|l|l|l|l|}
\hline \multirow{2}{*}{ Dataset } & \multicolumn{2}{|c|}{ mean error } & $\begin{array}{l}\text { mean error } \\
\text { (anomalies) }\end{array}$ \\
\cline { 2 - 4 } & SP & DP & SP \\
\hline Credit Fraud & $1.1127 \mathrm{E}-21$ & 0.4750 & $1.1127 \mathrm{E}-21$ \\
\hline APS Trucks & $2.9719 \mathrm{E}-13$ & 0.4750 & $2.9719 \mathrm{E}-13$ \\
\hline Synthetic & $3.2173 \mathrm{E}-5$ & 0.4750 & $3.2173 \mathrm{E}-5$ \\
\hline Mammography & 0.0022 & 0.4749 & 0.0021 \\
\hline Thyroid & 0.0870 & 0.4750 & 0.0867 \\
\hline
\end{tabular}

Table 2: effect of sparsity of databases. "mean error" is over the randomly picked $n$ records from the possible values of the records for each dataset for SP and DP mechanisms for $(\beta, r)$-AIQ. "mean error (anomalies)" is only over the anomalous records in the $n$ picked records. Here, $n$ is $20 \%$ of the size of the dataset and $\varepsilon=0.1$.

are provided by the domain experts [35]. Here, we employed the protocol outlined in Appendix A.2 to find $\beta$ and $r$; this protocol follows the basic idea of parameter selection presented in the work [35] that proposed the notion of $(\beta, r)$-anomaly. Table 1 gives the values of $\beta$ and $r$, which we found through the protocol, along with the number of true $(\beta, r)$-anomalies (true anomalies identifiable by $(\beta, r)$-anomaly method for the given parameter values).

Error: We measure the error of a private mechanism (which is a randomized algorithm) as its probability of outputting the wrong answer-recall that in the case of AIQ, there are only two possible answers, i.e. 0 or 1 . For each AIQ for a fixed record, we estimate the error by the average number of mistakes over $m$ trials. So for our experiments we choose $m$ to be 10000 .

For each dataset, we find all the true $(\beta, r)$-anomalies and for each of them perform private anomaly identification query using SP-mechanism (given in Section 4.1.2) and DP-mechanism (given in Section 4.1.1) for $\varepsilon=0.01,0.1$, and 1 and compute the error, which we give by the box plot in Figure 6. The reason we only considered our DP mechanism for this part is that it is the best among the baselines (see Table 3) and it also has strong accuracy guarantees (Theorem 4.4). The error of SP-mechanism, in many cases, is so small (e.g. of the order $10^{-15}$ or even smaller for larger values of $\varepsilon$ ) that it can be considered zero for all practical purposes. Furthermore, as the data size increases (and correspondingly the value of $\beta$ ), the error of SP-mechanism reduces. However, in the case of anomalies, the error of DP-mechanism is consistently close
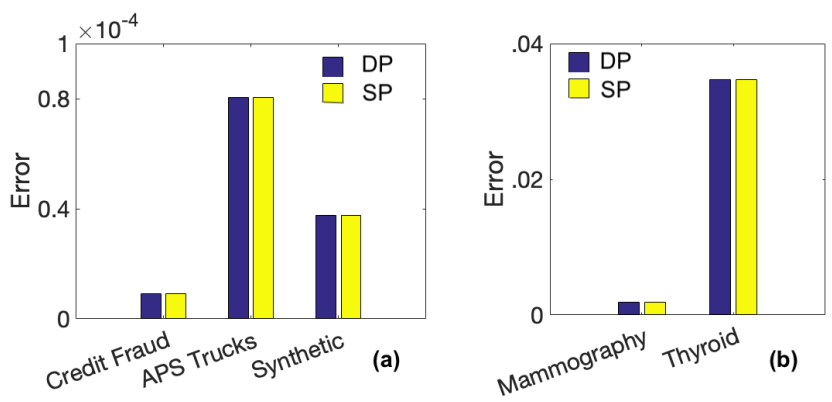

Figure 8: evaluation over normal records. (a),(b), give the average error of SP and DP mechanism for AIQ over all the normal records from each data set; $\varepsilon=0.1$.
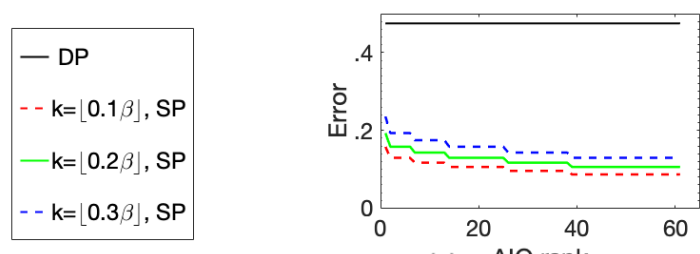

(a) AIQ rank

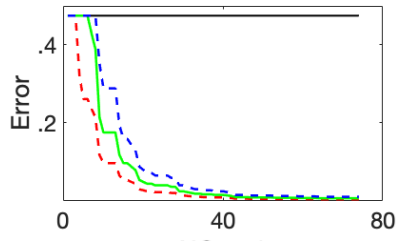

(b) AIQ rank

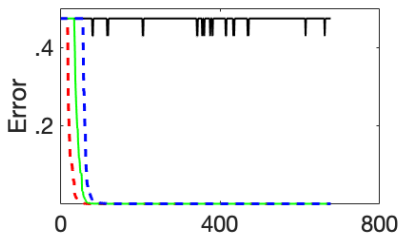

(d) AIQ rank

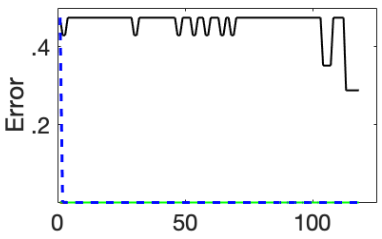

(c) AIQ rank

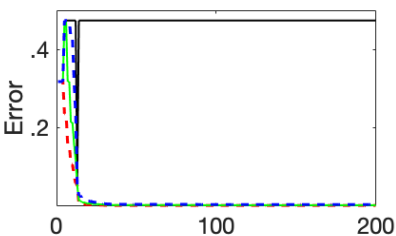

(e) AIQ rank

Figure 9: evaluation over true $(\beta, r)$-anomalies for varying $k$. (a)-(e), give the errors of SP and DP mechanisms. AIQ rank is given by the error of SP-mechanism for each anomaly: the higher the rank, the lower the error. Mechanisms are as given in Section 4 and $\varepsilon=1$. (a), Thyroid, (b), Mammography, (c), Credit Fraud, (d), APS Trucks, (e), Synthetic data.

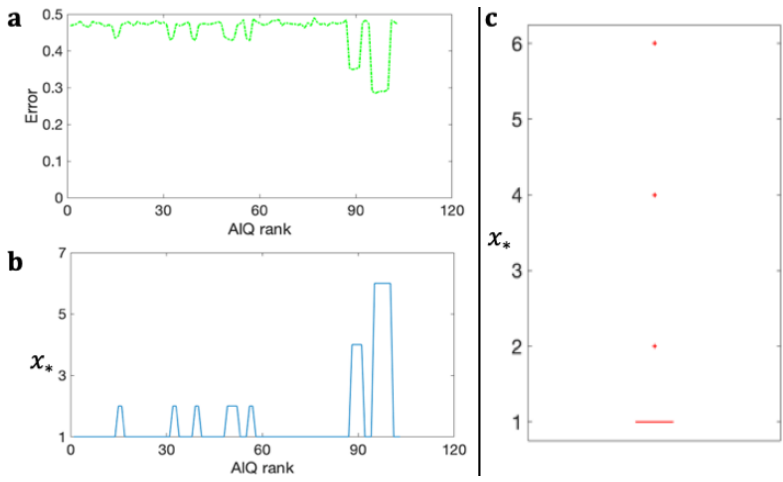

Figure 10: deviation in the DP-mechanism error for the Credit Fraud dataset. In (a), the plot is the same as given in Figure 9c for the DP-mechanism. In (b) and (c), $x_{*}$ for each record is the number of records in the database $x$ that have the same value. (c), shows the box plot for the data.

to that of random coin flip (i.e. selecting 0 or 1 with probability $1 / 2$ ) except for a few anomalous records in some cases - we will shortly explain the reason for this. The error of the SP-mechanism was overwhelmingly concentrated about zero (Figure 6), which is also true for the smaller values of $\varepsilon$. Thus, we can have higher privacy guarantee for sensitive records, while still being able to accurately identify anomalies. Also, note that as the size of the dataset increases, not only does the error of SP-mechanism reduces (for anomalies), 


\begin{tabular}{|c|c|c|c|c|c|c|c|c|c|c|c|c|}
\hline \multirow[t]{2}{*}{ Dataset } & \multicolumn{4}{|c|}{ Precision } & \multicolumn{4}{|c|}{ Recall } & \multicolumn{4}{|c|}{$F_{1}$-score } \\
\hline & $\mathrm{B}_{1}$ & $\mathrm{~B}_{2}$ & $\mathrm{DP}$ & SP & $\mathrm{B}_{1}$ & $\mathrm{~B}_{2}$ & DP & SP & $\mathrm{B}_{1}$ & $\mathrm{~B}_{2}$ & DP & SP \\
\hline Credit Fraud & 0.0101 & 0.0230 & 0.9930 & 0.9963 & 1.0000 & 0.0498 & 0.5250 & 0.9968 & 0.0199 & 0.0315 & 0.6868 & 0.9966 \\
\hline APS Trucks & 0.0115 & 0.0165 & 0.9870 & 0.9931 & 1.0000 & 0.0753 & 0.5263 & 0.9954 & 0.0227 & 0.0271 & 0.6865 & 0.9943 \\
\hline Synthetic & 0.0101 & 0.0114 & 0.9930 & 0.9963 & 1.0000 & 0.1189 & 0.5250 & 0.9968 & 0.0199 & 0.0208 & 0.6868 & 0.9966 \\
\hline Mammography & 0.0070 & 0.0081 & 0.0211 & 0.2004 & 0.8244 & 0.1000 & 0.5250 & 0.9977 & 0.0138 & 0.0149 & 0.0435 & 0.3337 \\
\hline Thyroid & 0.0174 & 0.0191 & 0.1427 & 0.3100 & 0.6656 & 0.2918 & 0.5250 & 0.8993 & 0.0339 & 0.0358 & 0.2244 & 0.4610 \\
\hline
\end{tabular}

Table 3: $B_{1}$ and $B_{2}$ are the best mechanisms from two families of mechanism. DP and SP are the mechanisms from Section 4.1.1 and Section 4.1.2 respectively. Going from red to blue the value decreases. $\varepsilon=0.1$

but also its divergence. Thus, it indicates that our methodology is even more appropriate for big data settings. On the other hand, for anomalies, the errors of DP-mechanism are concentrated about $1 /\left(1+e^{\varepsilon}\right)$ (Figure 7$)$. This is in accordance with our theoretical results and the assumption that the databases are typically sparse.

Next, we evaluated the performance over the normal records. Here, both the SP and the DP mechanisms performed equally (Figure 8). For the same value of $\varepsilon$, every sensitive record in the database has the same level of privacy under sensitive privacy as all the records under differential privacy; thus the same level of accuracy should be achievable under both the privacy notions. Here we see again that datasets with larger sizes exhibit very small error.

To evaluate the performance over future queries, we picked $n$ records uniformly at random from the space of possible (values of) records for each dataset $-n$ was set to be $20 \%$ of the size of the dataset. Here too the SP-mechanism outperforms the DPmechanism significantly (Table 2). This is because most of the randomly picked records are anomalous as per the $(\beta, r)$-anomaly, which is due to the sparsity of the databases. This fact becomes very clear when we compare the mean error over the random records to the mean error over the anomalous records in the randomly picked records (see the second and the last column of Table 2). Since the probability of observing a mistake is extremely small (e.g., 1 in $10^{10}$ trials), in Table 2, the mean is computed over the actual probability of error of the mechanism instead of the estimated error.

We already saw that by increasing $k$ we move the boundary between sensitive and non-sensitive records (Figure 3). So to observe the effect of varying values of $k$ on real world datasets, we carried out experiments on the datasets with $k=\lfloor 0.1 \beta\rfloor,\lfloor 0.2 \beta\rfloor$, and $\lfloor 0.3 \beta\rfloor$ - recall that a record is considered $k$-sensitive with respect to a database if the record is normal or becomes normal under the addition and (or) deletion of at most $k$ records from the database. Note that if $k \geq \beta+1$ then every record will be sensitive regardless of the database. The results are provided in Figure 9. Here we conclude that even for the higher values of $k$ SP-mechanism performs reasonably well. Further, if the size of dataset is large enough, then the loss in accuracy for most of the records is negligible.

We see that for Credit Fraud and APS Trucks datasets, differentially private AIQ for some of the anomalous records give smaller error. We explain this deviation using the Credit Fraud dataset as an example. The above mentioned deviation in the error occurs whenever the anomalous record is not unique (Figure 10a-b), which is typically rare (Figure 10c). The reason DP-mechanism's error remains constant in most cases is that the anomalies lie in a very sparse region of space and mostly do not have any duplicates (i.e., other records with the same value $-x_{i} \approx 1$ ).
Finally, to evaluate the overall performance of our SP-mechanism, we computed precision, recall, and $F_{1}$-score [1]. We also provide a comparison with two different baseline mechanisms, $B_{1}, B_{2}$ in addition to pareto optimal DP mechanism (see Table 3).

$B_{1}$ and $B_{2}$ are the best performing mechanisms (i.e., with the highest $F_{1}$-score) from two families of mechanisms. Each mechanism in each of the family is identified by a threshold $t$, where $0 \leq t \leq 1$. Below, we describe the mechanisms from both the families for fixed $\varepsilon$, threshold $t$, record $i \in \mathcal{X}$, and database $x \in \mathcal{D}$. The mechanism in the first family is given as $B_{1, t}(x)=1$ if and only if $O(x)+\operatorname{Lap}(1 / \varepsilon)>t \times\left(\|x\|_{1}+\operatorname{Lap}(1 / \varepsilon)\right)$; here $O(x)$ gives the number of anomalies in $x$ and $\operatorname{Lap}(1 / \varepsilon)$ is independent noise from Laplace distribution of mean zero and scale $1 / \varepsilon$. The mechanism in the second family is given as $B_{2, t}(x)=1$ if and only if $O(x)+\operatorname{Lap}(\beta / \varepsilon)>t \times\left(\|x\|_{1}+\operatorname{Lap}(1 / \varepsilon)\right)$. Note that, the mechanism from the first family are $\varepsilon_{1}$-DP, where $\varepsilon_{1} \geq \beta \varepsilon$. This is due to the fact that $\max _{x, y \in \mathcal{D}:\|x-y\|_{1}=1}|O(x)-O(y)|=\beta[15]$. However the mechanism from the second family are $\varepsilon_{2}$-DP, where $\varepsilon_{2} \geq \varepsilon$.

Our mechanism outperforms all the baselines. Furthermore, DPmechanism largely outperforms the rest of the baselines.

\section{RELATED WORK}

To our knowledge, there has been no work that formally explores the privacy-utility trade-off in privately identifying anomalies, where sensitive records (which include the normal records defined in a data-dependent fashion) are protected against inference attacks about their presence or absence in the database used.

Differential privacy $[15,17]$ has shaped the field of private data analysis. This notion aims to protect everyone, and in a sense, many of the DP mechanisms (e.g. Laplace mechanism) achieve privacy by protecting anomalies; and in doing so perturb the information regarding anomalies greatly. This adversely affects the accuracy of anomaly detection and identification. Furthermore, differential privacy is a special case of sensitive privacy (Section 4.1.1).

Variants of the notion of differential privacy address important practical challenges. In particular, personalized differential privacy [29], protected differential privacy [31], relaxed differential privacy [6], and one-sided differential privacy [13] have a reversed order of quantification compared to sensitive privacy. Sensitive privacy, quantifies sensitive records and their privacy after quantifying the database, which is in contrast to the previous work. Thus, under sensitive privacy, it is possible for a record of some value to be sensitive in one database and not in the other, while this cannot be the case in the above mentioned definitions. On the other hand, by labeling records independent to the database (as in the previous work) one can solve a range of privacy problems such as counting 
queries and releasing histograms. Hence, this work solves the open problem (in [31]). Next, we present an individual comparison with each of the above mentioned previous work along with some other relevant ones from the literature.

Protected differential privacy [31] proposes an algorithm for social networks to search for anomalies that are fixed and are defined independent of the database. This is not extensible to the case, where anomalies are defined relative to the other records [31]. Similarly, the proposed relaxed DP mechanism [6] is only applicable to anomalies defined in data-independent manner.

One-sided differential privacy (OSDP) [13] is a general framework, and is useful for the applications, where one can define the records to be protected independent of the database. Note that the notion of sensitive record in OSDP is different from the one considered here. Further, due to its asymmetric nature of the privacy constrains, OSDP fails to protect against the inference about the presence/absence of a sensitive record (in general), which is not the case with sensitive privacy (see Appendix A.10.1).

Tailored differential privacy (TDP) [36] provides varying levels of privacy for a record, which is given by a function, $\alpha$, of the record's value and the database. However, the work is restricted to releasing histograms, where outliers are provided more privacy. Whereas our focus is identifying anomalies, where anomalies may have lesser privacy. Further, the notion of anomaly used in the work [36] is the simple $(\beta, 0)$-anomaly. Extending it to the case of $r>0$ is a non-trivial task since, here, changing a record in the database may affect the label (outlyingness) of another record with a different value. We also note that sensitive privacy is a specialized case of tailored differential privacy (see Appendix A.10.2.)

Blowfish privacy (BP) [25] and Pufferfish privacy (PP) [33] are general frameworks, and provide no concrete methodology or direction to deal with anomaly detection or identification, where anomalies are defined in a data-dependent fashion. Sensitive privacy is a specialized class of definitions under these frameworks.

Thus, in term of definition, our contribution in comparison with OSDP [13], TDP [36], BP [25], and PP [33], is defining the the notion of sensitive record and the sensitive neighborhood graph that is appropriate and meaningful for anomalies (when defined relative to the other records) and giving constructions and mechanisms for identifying anomalies.

Finally, [4] proposed a method for searching outliers, which can depend on data, but this is done in a rather restricted setting, which has theoretical value (in [4] the input databases are guaranteed to have only one outlier, a structure not present in the typical available datasets; this is in addition to other input database restrictions required by [4]).

Other relaxations of differential privacy such as [2] is specifically for location privacy and [16] is to achieve fairness in classification to prevent discrimination against individuals based on their membership in some group and as such are not applicable to the problem we consider here.

\section{KEY TAKEAWAYS AND CONCLUSION}

This work is the first to lay out the foundations of the privacypreserving study of data dependent anomalies and develop general constructions to achieve this. It is important to reiterate that the formalization and conceptual development is independent of any particular definition of anomaly. Indeed, the definition of sensitive privacy (Definitions 3.1 and 3.2), and the constructions to achieve it (Construction 1 and Construction 2) are general and work for an arbitrary definition of anomaly (Theorem 4.1 and Theorem 4.7).

We noted earlier that sensitive privacy generalizes differential privacy. Thus, the guarantees provided by sensitive privacy are similar to that of differential privacy, and in fact, Construction 1 can be employed to give differentially private mechanisms for computing anomaly identification query or any binary function. However, in general, the guarantee provided by sensitive privacy to any two databases differing by one record could be correspondingly weaker than that offered by differential privacy depending on the distance between the databases in the sensitive neighborhood graph. There is also a divergence in guarantees in terms of composition. In differential privacy, composition is only in terms of the privacy parameter, $\varepsilon$. However, for sensitive privacy, composition needs to take into account not only the privacy parameter $\varepsilon$, but also the sensitive neighborhood graphs corresponding to the queries being composed. Nevertheless, the composition and post-processing properties (Section 3.1) hold regardless of the notion of the anomaly.

An extensive empirical study carried out over data from diverse domains overwhelmingly supports the usefulness of our method. The sensitively private mechanism consistently outperforms differentially private mechanism with exponential gain in accuracy in almost all cases. Although it is easy to come up with example datasets where a differentially private mechanism also performs well (e.g., $(\beta, r)$-AIQ for $i$ and $x$ when $\left.x_{i}=B_{x}(i, r)=\beta / 2\right)$, the experiments with real data show that such cases are unlikely to occur in practice. Indeed, the experiments show that most of the anomalies occur in the setting, where an $\varepsilon$-DP mechanism performs the worst, that is, its error is close to $1 /\left(1+e^{\varepsilon}\right)$ (a lower bound on the error of any $\varepsilon$-DP mechanism and follows from Claim 1 ).

To conclude, in this paper, we develop methods for anomaly identification that provide a provable privacy guarantee to all records, which is calibrated to their degree of being anomalous (in a datadependent sense), while enabling the accurate identification of anomalies. We stress that the currently available methodologies for protecting privacy in data analysis are fundamentally unsuitable for the task at hand: they either fail to stop identity inference from the data, or lack the ability to deal with the data-dependent definition of anomaly. Note that anomaly identification is only the first step to tackling the problem of anomaly detection (finding all the anomalous records in a dataset). In the future, we plan to tackle this and instantiate our framework for other anomaly detection models.

\section{ACKNOWLEDGMENTS}

Research reported in this publication was supported by the National Science Foundation under awards CNS-1422501, CNS-1564034, CNS1624503, CNS-1747728 and the National Institutes of Health under award R01GM118574. The content is solely the responsibility of the authors and does not necessarily represent the official views of the agencies funding the research.

\section{REFERENCES}

[1] Charu C Aggarwal. 2015. Outlier analysis. In Data mining. Springer, 237-263. 
[2] Miguel E Andrés, Nicolás E Bordenabe, Konstantinos Chatzikokolakis, and Catuscia Palamidessi. 2013. Geo-indistinguishability: Differential privacy for locationbased systems. In Proceedings of the 2013 ACM SIGSAC conference on Computer \& communications security. ACM, 901-914.

[3] Vic Barnett and Toby Lewis. 2000. Outliers in statistical data. Wiley.

[4] Daniel M Bittner, Anand D Sarwate, and Rebecca N Wright. 2018. Using Noisy Binary Search for Differentially Private Anomaly Detection. In International Symposium on Cyber Security Cryptography and Machine Learning. Springer, $20-37$.

[5] Martin Bobrow. 2013. Balancing privacy with public benefit. Nature News 500 7461 (2013), 123.

[6] Jonas Böhler, Daniel Bernau, and Florian Kerschbaum. 2017. Privacy-preserving outlier detection for data streams. In IFIP Annual Conference on Data and Applications Security and Privacy. Springer, 225-238.

[7] Centers for Medicare \& Medicaid Services. 1996. The Health Insurance Portability and Accountability Act of 1996 (HIPAA). Online at http://www.cms.hhs.gov/hipaa/.

[8] Varun Chandola, Arindam Banerjee, and Vipin Kumar. 2009. Anomaly detection: A survey. ACM computing surveys (CSUR) 41, 3 (2009), 15.

[9] Ronald Cramer, I. B. DamgÃerrd, and Jesper Buus Nielsen. 2015. Secure multiparty computation: an information-theoretic approach. Cambridge University Press.

[10] Andrea Dal Pozzolo, Olivier Caelen, Reid A Johnson, and Gianluca Bontempi. 2015. Calibrating probability with undersampling for unbalanced classification. In Computational Intelligence, 2015 IEEE Symposium Series on. IEEE, 159-166.

[11] Alison M Darcy, Alan K Louie, and Laura Weiss Roberts. 2016. Machine learning and the profession of medicine. Jama 315, 6 (2016), 551-552.

[12] Yihe Dong, Samuel B Hopkins, and Jerry Li. 2019. Quantum Entropy Scoring for Fast Robust Mean Estimation and Improved Outlier Detection. arXiv preprint arXiv:1906.11366 (2019).

[13] Stelios Doudalis, Ios Kotsogiannis, Samuel Haney, Ashwin Machanavajjhala, and Sharad Mehrotra. 2017. One-sided differential privacy. arXiv preprint arXiv:1712.05888 (2017).

[14] Dheeru Dua and Casey Graff. 2017. UCI Machine Learning Repository. http: //archive.ics.uci.edu/ml

[15] Cynthia Dwork. 2006. Differential Privacy. In Automata, Languages and Programming, Michele Bugliesi, Bart Preneel, Vladimiro Sassone, and Ingo Wegener (Eds.). Springer Berlin Heidelberg, Berlin, Heidelberg, 1-12.

[16] Cynthia Dwork, Moritz Hardt, Toniann Pitassi, Omer Reingold, and Richard Zemel. 2012. Fairness through awareness. In Proceedings of the 3rd innovations in theoretical computer science conference. ACM, 214-226.

[17] Cynthia Dwork, Frank McSherry, Kobbi Nissim, and Adam Smith. 2006. Calibrating noise to sensitivity in private data analysis. In TCC. Springer, 265-284.

[18] Cynthia Dwork, Aaron Roth, et al. 2014. The algorithmic foundations of differential privacy. Foundations and Trends ${ }^{\circledR}$ in Theoretical Computer Science 9, 3-4 (2014), 211-407.

[19] Cynthia Dwork, Adam Smith, Thomas Steinke, Jonathan Ullman, and Salil Vadhan 2015. Robust traceability from trace amounts. In Foundations of Computer Science (FOCS), 2015 IEEE 56th Annual Symposium on. IEEE, 650-669.

[20] Yaniv Erlich and Arvind Narayanan. 2014. Routes for breaching and protecting genetic privacy. Nature Reviews Genetics 15, 6 (2014), 409.

[21] 2016. Regulation (EU) 2016/679 of the European Parliament and of the Council of 27 April 2016 on the protection of natural persons with regard to the processing of personal data and on the free movement of such data, and repealing Directive 95/46/EC (General Data Protection Regulation). Official fournal of the European Union L119 (4 May 2016), 1-88. http://eur-lex.europa.eu/legal-content/EN/TXT/ ?uri=OJ:L:2016:119:TOC

[22] David Freedman, Robert Pisani, and Roger Purves. 1998. Statistics. W.W. Norton.

[23] Machine Learning Group. 2018. Credit Card Fraud Detection. https://www. kaggle.com/mlg-ulb/creditcardfraud/home.

[24] Melissa Gymrek, Amy L McGuire, David Golan, Eran Halperin, and Yaniv Erlich. 2013. Identifying personal genomes by surname inference. Science 339, 6117 (2013), 321-324.

[25] Xi He, Ashwin Machanavajjhala, and Bolin Ding. 2014. Blowfish privacy: Tuning privacy-utility trade-offs using policies. In Proceedings of the 2014 ACM SIGMOD. ACM, 1447-1458.

[26] Nils Homer, Szabolcs Szelinger, Margot Redman, David Duggan, Waibhav Tembe Jill Muehling, John V Pearson, Dietrich A Stephan, Stanley F Nelson, and David W Craig. 2008. Resolving individuals contributing trace amounts of DNA to highly complex mixtures using high-density SNP genotyping microarrays. PLoS genetics 4, 8 (2008), e1000167.

[27] Marcello Ienca, Pim Haselager, and Ezekiel J Emanuel. 2018. Brain leaks and consumer neurotechnology. Nature biotechnology 36, 9 (2018), 805-810.

[28] Ian Jolliffe. 2011. Principal component analysis. In International encyclopedia of statistical science. Springer, 1094-1096.

[29] Zach Jorgensen, Ting Yu, and Graham Cormode. 2015. Conservative or liberal? personalized differential privacy. In 2015 IEEE 31st International Conference on Data Engineering (ICDE). IEEE, 1023-1034.
[30] Seppo Karrila, Julian Hock Ean Lee, and Greg Tucker-Kellogg. 2011. A comparison of methods for data-driven cancer outlier discovery, and an application scheme to semisupervised predictive biomarker discovery. Cancer informatics 10 (2011), CIN-S6868.

[31] Michael Kearns, Aaron Roth, Zhiwei Steven Wu, and Grigory Yaroslavtsev. 2016. Private algorithms for the protected in social network search. Proceedings of the National Academy of Sciences 113, 4 (2016), 913-918.

[32] Daniel Kifer and Bing-Rong Lin. 2012. An axiomatic view of statistical privacy and utility. Fournal of Privacy and Confidentiality 4, 1 (2012), 5-49.

[33] Daniel Kifer and Ashwin Machanavajjhala. 2014. Pufferfish: A framework for mathematical privacy definitions. ACM Transactions on Database Systems (TODS) 39,1 (2014), 3.

[34] Edwin M Knorr and Raymond T Ng. 1997. A Unified Notion of Outliers: Properties and Computation.. In KDD, Vol. 97. 219-222.

[35] Edwin M Knorr and Raymond T Ng. 1998. Algorithms for mining distancebased outliers in large datasets. In Proceedings of the 1998 VLDB. Citeseer, 392-403.

[36] Edward Lui and Rafael Pass. 2015. Outlier privacy. In TCC. Springer, 277-305.

[37] D Luquetti, P Claes, DK Liberton, K Daniels, KM Rosana, EE Quillen, LN Pearson, B McEvoy, M Bauchet, AA Zaidi, et al. 2014. Modeling 3D Facial Shape from DNA. PLoS Genetics 10, 3 (2014), e1004224.

[38] Ye Nan, Kian Ming Chai, Wee Sun Lee, and Hai Leong Chieu. 2012. Optimizing F-measure: A Tale of Two Approaches. Proceedings of the 29th International Conference on Machine Learning, ICML 20121 (06 2012).

[39] Ziad Obermeyer and Ezekiel J Emanuel. 2016. Predicting the futureâĂŤbig data, machine learning, and clinical medicine. The New England journal of medicine 375, 13 (2016), 1216.

[40] Soumi Ray, Dustin S McEvoy, Skye Aaron, Thu-Trang Hickman, and Adam Wright. 2018. Using statistical anomaly detection models to find clinical decision support malfunctions. Fournal of the American Medical Informatics Association (2018).

[41] Shebuti Rayana. 2016. ODDS Library. http://odds.cs.stonybrook.edu Available at http://odds.cs.stonybrook.edu.

[42] Gordon D Schiff, Lynn A Volk, Mayya Volodarskaya, Deborah H Williams, Lake Walsh, Sara G Myers, David W Bates, and Ronen Rozenblum. 2017. Screening for medication errors using an outlier detection system. Fournal of the American Medical Informatics Association 24, 2 (2017), 281-287.

\section{A APPENDIX}

\section{A.1 Empirical evaluation protocols}

Evaluation over normally distributed data: If the data is from one dimensional normal distribution with mean $\mu$ and standard deviation $\sigma$ then a record $i$ is anomalous (or equivalently an outlier) if $|i-\mu| \geq 3 \sigma$, and is statistically equivalent to $\left(\beta=1.2 \times 10^{-3} n, r=\right.$ $0.13 \sigma)$-anomaly [35], where $n$ is the size of the database.

To adapt this result for 2D normal distribution in Figure 2, set $r=0.13 \sqrt{\sigma_{1}^{2}+\sigma_{2}^{2}}$ and compute $\beta$ in a similar fashion as above. Next, take 30 samples of size $20 K$, i.e. $n=20,000$, from the $2 \mathrm{D}$ normal distribution, $N(\mu, \Sigma)$, where $\mu=(0,0)$ and $\Sigma=\left[\begin{array}{ll}2 & 0 \\ 0 & 2\end{array}\right]$, and run SP-mechanism (given in Section 4.1.2) and DP-mechanism (given in Section 4.1.1) for $(\beta, r)$-anomaly identification query to compute accuracy, which is measured by the probability of outputting the correct answer by the private mechanism, and average the results over the samples for each query. We then plot the average accuracy and interpolate the results using one-degree polynomial in the two coordinates (Figure 2b-c). We used the "ListPlot3D" function of Mathematica with the argument "InterpolationOrder" set to 1 .

In Figure 2d-e and Figure 3, we plot the level of privacy (in term of $\varepsilon)$ that each record (point) has under private anomaly identification query. Here, the level of privacy for a record in a given database is measured by the maximum divergence divergence in the probability of outputting a label when we add or remove the record from the database. For $\varepsilon$-SP-mechanism, $U$, to compute the value of the privacy parameter, $\varepsilon$, for a record $i$ in a given database $x$, consider databases $y$ and $z$. $y$ and $z$ are same as $x$ except for $y$ has one more record of value $i$ and $z$ has one less record of value $i$-if there is 
no record of value $i$ in $x$ then $z$ will be the same as $x$. Now we can calculate $e^{\varepsilon}$ for record $i$ be by (4).

$$
e^{\varepsilon}=\max _{w \in\{y, z\}} \max _{b \in\{0,1\}}\left(\frac{P(U(x)=b)}{P(U(w)=b)}, \frac{P(U(w)=b)}{P(U(x)=b)}\right)
$$

\section{A.2 Protocol for $(\beta, r)$ selection}

The main idea is to fix a value of $\beta$, for a dataset of size $n$, as $(1-p) \times n$, where $p$ is close to 1 , and then search for an appropriate value of $r$. It is recommended [35] that for the datasets of sizes $10^{3}$ and $10^{6}, \beta$ be $(1-0.995) \times 10^{3}$ and $(1-0.99995) \times 10^{6}$. By assuming that $p$ is linearly related to $n$, one can use the provided values to find the value of $\beta$ for any given dataset. For a fixed value of $\beta$, a search is performed to find $r$ that maximize the $F_{1}$-score (also known as balanced $F$-measure), which is a popular performance metric for imbalanced datasets [38], and it is the harmonic mean of precision and recall. We used the following protocol to select the value of $r$. Initialize $r_{\min }=.001, r_{\max }=40$ (or the value that is not smaller than the maximum distance between any two points in the given dataset), $r=0$, and $S=0$. Next, set $r_{1}=r_{\min }+\left(r_{\max }-r_{\min }\right) / 4, r_{2}=$ $r_{\min }+3\left(r_{\max }-r_{\min }\right) / 4$, pick $\alpha$ from $[0,1]$ uniformly at random and set $\mathrm{r}_{3}=\alpha r_{1}+(1-\alpha) r_{2}$. Compute $F_{1}$-score for each of the $r^{\prime} s$, i.e. $S_{r_{1}}, S_{r_{2}}$, and $S_{r_{3}}$. Let $S_{r_{t}}$ be the maximum of the computed scores. If $S_{r_{t}}$ is greater than $S$ then set $S=S_{r_{t}}$ and $r=r_{t}$; further, if $S_{r_{2}}<S$ and $r_{2}>r$ then set $r_{\max }=r_{2}$ but if it is not the case and $S_{r_{1}}<S$ and $r_{1}<r$ then set $r_{\min }=r_{1}$, otherwise do nothing. Repeat this process, except for the initialization step, until the improvement in $\mathrm{S}$ becomes insignificant. In our experiments, repeating the process for ten iterations generally sufficed.

\section{A.3 Proof of Claim 1}

Proof. Arbitrarily fix $\varepsilon>0, f: \mathcal{D} \rightarrow\{0,1\}, \varepsilon$-differentially private mechanism $M: \mathcal{D} \rightarrow\{0,1\}$, and $x, y \in \mathcal{D}$ such that $f(x) \neq$ $f(y)$ and $\|x-y\|_{1}=1$; and let $b=f(x)$.

If $\mathrm{P}(M(y)=b) \leq 1 /\left(1+e^{\varepsilon}\right)$ then, by differential privacy constraints, we get that $\mathrm{P}(M(x)=b) \leq e^{\varepsilon} /\left(1+e^{\varepsilon}\right)$; thus $\mathrm{P}(M(x)=1-b) \geq$ $1 /\left(1+e^{\varepsilon}\right)$. Similarly, $\mathrm{P}(M(x)=1-b) \leq 1 /\left(1+e^{\varepsilon}\right)$ implies $\mathrm{P}(M(y)=b) \geq$ $1 /\left(1+e^{\varepsilon}\right)$. Hence, from the above, it follows that

$$
\max (\mathrm{P}(M(x) \neq f(x)), \mathrm{P}(M(y) \neq f(y))) \geq 1 /\left(1+e^{\varepsilon}\right)
$$

Since $M, x$ and $y$ were fixed arbitrarily, the claim follows, and this completes the proof.

\section{A.4 Proof of Theorem 4.1}

Proof. Fix arbitrary $\varepsilon>0$ and a definition of anomaly. Let $g$ be the anomaly identification function and $G_{S}$ be the $k$-sensitive neighborhood graph corresponding to it for an arbitrary value of $k \geq 1$. Fix $\lambda$ to be 1-Lipschitz continuous lower bound on the mdd-function, $\Delta_{G_{S}}$, for $g$ such that $\lambda \geq 1$. Let $U_{\lambda}$ be as given by Construction 1 . Next, fix an anomaly identification query, $(i, g)$, and $x, y \in \mathcal{D}$ that are neighbors (i.e. connected by a direct edge) in $G_{S}$.

$$
\begin{aligned}
& \text { If } g(i, x)=g(i, y)=b \text { from some } b \in\{0,1\} \text { then } \\
& \qquad \begin{aligned}
\frac{\mathrm{P}\left(U_{\lambda}(x) \neq b\right)}{\mathrm{P}\left(U_{\lambda}(y) \neq b\right)} & =e^{\varepsilon(-\lambda(i, x)+\lambda(i, y))} \\
& \leq e^{\varepsilon|-\lambda(i, x)+\lambda(i, y)|} \\
& \leq e^{\varepsilon} \quad \text { (since } \lambda \text { is 1-Lipschitz continuous) } \\
\text { and } & \\
\frac{\mathrm{P}\left(U_{\lambda}(x)=b\right)}{\mathrm{P}\left(U_{\lambda}(y)=b\right)} & =\frac{1-\mathrm{P}\left(U_{\lambda}(x) \neq b\right)}{1-\mathrm{P}\left(U_{\lambda}(y) \neq b\right)} \\
& =\frac{1+e^{\varepsilon}-e^{-\varepsilon(\lambda(i, x)-1)}}{1+e^{\varepsilon}-e^{-\varepsilon(\lambda(i, y)-1)}} \\
& =\frac{e^{\varepsilon \lambda(i, y)}\left(1+e^{\varepsilon}\right)-e^{\varepsilon(\lambda(i, y)-\lambda(i, x)+1)}}{e^{\varepsilon \lambda(i, y)}\left(1+e^{\varepsilon}\right)-e^{\varepsilon}}
\end{aligned}
\end{aligned}
$$

since $\lambda$ is 1 -Lipschitz continuous, it follows that

$$
\frac{\mathrm{P}\left(U_{\lambda}(x)=b\right)}{\mathrm{P}\left(U_{\lambda}(y)=b\right)} \leq \frac{e^{\varepsilon \lambda(i, y)}\left(1+e^{\varepsilon}\right)-1}{e^{\varepsilon \lambda(i, y)}\left(1+e^{\varepsilon}\right)-e^{\varepsilon}} \leq \frac{\left(1+e^{\varepsilon}\right)-1}{\left(1+e^{\varepsilon}\right)-e^{\varepsilon}}=e^{\varepsilon}
$$

The first inequality holds because $\lambda$ is 1-Lipschitz continuous, and the second one holds since $\lambda \geq 1$.

On the other hand, if $g(i, x) \neq g(i, y)$, then $\lambda(i, x)=\lambda(i, y)=1$. This holds because $x$ and $y$ are neighbors, i.e. $d_{G_{S}}(x, y)=1$, and hence, $\Delta_{G_{S}}(i, x)=\Delta_{G_{S}}(i, y)=1$ and $\lambda$ is such that $\Delta_{G_{S}}(j, z) \geq$ $\lambda(j, z) \geq 1$ for every $j \in \mathcal{X}$ and $z \in \mathcal{D}$. Thus, in this case, the privacy constraints trivially hold. This concludes the formal argument.

\section{A.5 Proof of Lemma 4.3}

Proof. Let $\mathbb{G}$ be the neighborhood graph over $\mathcal{D}, d$ be the distance metric over $\mathcal{X} \times \mathcal{X}, d_{\mathbb{G}}$ be the shortest path length metric over $\mathbb{G}$, and $g$ be the anomaly identification function for $(\beta, r)$-anomaly for arbitrarily fixed values of $\beta \geq 1$ and $r \geq 0$.

Firstly, we prove that the $\Delta_{\mathbb{G}}$ given by (2) is indeed the mddfunction $(\beta, r)$-AIQ. Arbitrarily fix $i \in \mathcal{X}$ and any database $x \in \mathcal{D}$. We know that the value of $g(i, x)$ only depends upon $x_{i}$ and $B_{x}(i, r)-$ recall that $g(i, x)=1 \Longleftrightarrow x_{i} \geq 1$ and $B_{x}(i, r) \leq \beta$. Further, $d_{\mathbb{G}}(x, y)=\|x-y\|_{1}$ since every two databases that differ by exactly one record are directly connected by an edge. Hence, it follows that for $C_{i}=\{j \in \mathcal{X}: d(i, j) \leq r\}$,

$$
\Delta_{\mathbb{G}}(i, x)=\min _{y: g(i, y) \neq g(i, x)}\|x-y\|_{1}=\min _{y: g(i, y) \neq g(i, x)} \sum_{j \in C_{i}}\left|x_{j}-y_{j}\right| .
$$

We will consider four cases based on the condition (given in the $\Delta_{\mathbb{G}}$ ) that $x$ satisfies. From (5), we know that $\Delta_{\mathbb{G}}(i, x)$ is the same as the minimum number of records by which a database $y$ differs such that $g(i, x) \neq g(i, y)$. Thus in the proof we will modify the database $x$ by adding or (and) removing records from $x$, and show that minimum number of changes required in $x$ to change the output of $g$ is given by $\Delta_{\mathbb{G}}$.

Case 1: When $x$ satisfies the first condition, $g(i, x)=0$. For any database $y$ such that $g(i, y)=1$, it must hold that $y_{i} \geq 1$ and $B_{y}(i, r) \leq \beta$. So we obtain a $y$ by adding one record of value $i$ to $x$. Thus $\Delta_{\mathbb{G}}(i, x)=1$. 
Case 2: When $x$ satisfies the second condition, here again similar to the case above, $g(i, x)=0$, and for any database $y$ such that $g(i, y)=1$, it must hold that $y_{i} \geq 1$ and $B_{y}(i, r) \leq \beta$. So we will have to add one record of value $i$ to $x$ to obtain a database $y^{\prime}$, but now $B_{y^{\prime}}(i, r) \geq \beta+1$. Thus, to obtain a $y$, we will have to remove $B_{y^{\prime}}(i, r)-\beta=B_{x}(i, r)+1-\beta$ records of values in $C_{i} \backslash\{i\}$ from $y^{\prime}$ (or $x$ ). Thus, $\Delta_{\mathbb{G}}(i, x)=1+B_{x}(i, r)+1-\beta$.

Case 3: Here we assume that $x$ satisfies the third condition; hence $g(i, x)=1$. For a $y$ such that $g(i, y)=0$, either $y_{i}=0$ or $B_{y}(i, r) \geq$ $\beta+1$. Thus $\Delta_{\mathbb{G}}(i, x)$ will be the minimum of $x_{i}$ (which corresponds to the number of records of value $i$ present in $x$ that we will have to remove) and $\beta+1-B_{x}(i, r)$ (which corresponds to the number of records of values in $C_{i}$ that we will have to add to $x$ ).

Case 4: In this case, $g(i, x)=0$ because $B_{x}(i, r)>\beta$. Thus, we will have to remove $B_{x}(i, r)-\beta$ records of values in $C_{i}$ from $x$ such that there is at least on record of value $i$ in the modified $x$. Hence, $\Delta_{\mathbb{G}}(i, x)=B_{x}(i, r)-\beta$.

Further, in all the cases, $\Delta_{\mathbb{G}}(i, x) \geq 1$. Therefore, we conclude the $\Delta_{\mathbb{G}}$ is the mdd-function for $g$ (i.e. $(\beta, r)$-AIQ).

Next, we prove that the $\Delta_{\mathbb{G}}$ is 1 -Lipschitz continuous. Arbitrary fix $i$ and any two neighboring databases, $x$ and $y$ in $\mathbb{G}$. Let the $(k, l)$ represent that $x$ and $y$ respectively satisfy the $k^{t h}$ and $l^{t h}$ conditions in the $\Delta_{\mathbb{G}}$, where $k, l \in[4]$ such that $k \leq l$. We will prove that for each $(k, l)$, the $\Delta_{\mathbb{G}}$ satisfies the 1-Lipschitz continuity condition. Here, note that if the $\Delta_{\mathbb{G}}$ satisfies the 1-Lipschitz continuity condition under $(k, l)$ then it also satisfies the condition under $(l, k)$ because $\left|\Delta_{\mathbb{G}}(i, x)-\Delta_{\mathbb{G}}(i, y)\right|=\left|\Delta_{\mathbb{G}}(i, y)-\Delta_{\mathbb{G}}(i, x)\right|$.

For $(1,1),\left|\Delta_{\mathbb{G}}(i, x)-\Delta_{\mathbb{G}}(i, y)\right|=0$, and for $(2,2)$ and $(4,4)$, $\left|\Delta_{\mathbb{G}}(i, x)-\Delta_{\mathbb{G}}(i, y)\right| \leq 1$ since $\left|B_{x}(i, r)-B_{y}(i, r)\right| \leq 1$. Below, we consider rest of the cases.

$(3,3)$ : The case, when $B_{x}(i, r)=B_{y}(i, r)$, is trivial. So, let $B_{x}(i, r)=$ $1+B_{y}(i, r)$-this is without loss of generality since $\| x-$ $y \|_{1}=1$ and $\left|\Delta_{\mathbb{G}}(i, x)-\Delta_{\mathbb{G}}(i, y)\right|=\left|\Delta_{\mathbb{G}}(i, y)-\Delta_{\mathbb{G}}(i, x)\right|$. Thus, $\Delta_{\mathbb{G}}(i, x)=\min \left(x_{i}, \beta-B_{y}(i, r)\right)$ and $\Delta_{\mathbb{G}}(i, y)=\min \left(y_{i}, \beta+1-\right.$ $\left.B_{y}(i, r)\right)$. All the subcases, except for the following, trivially follow from $\|x-y\|_{1}=1$.

(a) $\Delta_{\mathbb{G}}(i, x)=x_{i}$ and $\Delta_{\mathbb{G}}(i, y)=\beta+1-B_{y}(i, r)$

(b) $\Delta_{\mathbb{G}}(i, x)=\beta-B_{y}(i, r)$ and $\Delta_{\mathbb{G}}(i, y)=y_{i}$

(a) is not possible as it requires $x_{i}<y_{i}$; this cannot happen because $\|x-y\|_{1}=1$ and $B_{x}(i, r)=1+B_{y}(i, r)$. As for $(b)$, the following holds for $t=\Delta_{\mathbb{G}}(i, x)-\Delta_{\mathbb{G}}(i, y)$ :

$-1 \leq \beta-B_{y}(i, r)-\left(\beta+1-B_{y}(i, r)\right) \leq t \leq x_{i}-y_{i} \leq 1$

Thus, it follows that 1-Lipschitz continuity condition is satisfied in this case.

$(1,2)$ : This happens when $B_{x}(i, r)=\beta-1$ and $B_{y}(i, r)=\beta$, which is sufficient for the condition to be satisfied.

$(1,3)$ : It is possible when $y_{i}=1$ and $1 \leq B_{y}(i, r) \leq \beta$; hence $\Delta_{\mathbb{G}}(i, y)=y_{i}$ and the case holds.

$(1,4)$ : This case is not possible since $\|x-y\|_{1}=1$, and the case requires $B_{x}(i, r)<\beta$ and $B_{y}(i, r)>\beta$.

$(2,3)$ : This case too is not possible since it requires $B_{x}(i, r) \geq$ $B_{y}(i, r)$ and $x_{i}<y_{i}$, when $\|x-y\|_{1}=1$.
$(2,4)$ : Here, $B_{x}(i, r)-B_{y}(i, r)=-1\left(\right.$ since $x_{i}=0$ and $\left.y_{i} \geq 1\right)$; hence the case follows.

$(3,4)$ : Here, it must hold that $B_{x}(i, r)=\beta$ and $B_{y}(i, r)=\beta+1$. Hence, $\Delta_{\mathbb{G}}(i, x)=1$ (since $\left.x_{i} \geq 1\right)$ and $\Delta_{\mathbb{G}}(i, y)=1$, and the case follows.

Since the $\Delta_{\mathbb{G}}$ satisfies 1-Lipschitz continuity condition under all the cases for arbitrary $i$ and arbitrary neighbors, $x$ and $y$, in the neighborhood graph, it holds for every $i$ and every two neighbors. Thus the claim follows. This completes the proof.

\section{A.6 Proof of Theorem 4.4}

Proof. Arbitrarily fix $\varepsilon$ and a $(\beta, r)$-AIQ, $(i, g)$. Let $\Delta_{\mathbb{G}}$ be as given by (2) and $U_{\Delta_{\mathbb{G}}}$ be as given by Construction 1 .

Firstly, note that $U_{\Delta_{\mathbb{G}}}$ is $\varepsilon$-DP. It follows from the facts that $\Delta_{\mathbb{G}} \geq$ 1, is 1-Lipschitz continuous (Lemma 4.3), and SP generalizes DP.

Next, we prove the optimality claim. We prove the claim using its contrapositive, that is, if there is a mechanism that is "better" than $U_{\Delta_{\mathbb{G}}}$, then it must not be $\varepsilon$-DP.

Assume there exits a DP mechanism $M$ such that for every $x, P\left(M(x)=g_{i}(x)\right) \geq P\left(U_{\Delta_{G}}(x)=g_{i}(x)\right)$ and for a database $y$, $P\left(M(y)=g_{i}(y)\right)>P\left(U_{\Delta_{\mathbb{G}}}(y)=g_{i}(y)\right)$ (i.e. $U_{\Delta_{\mathbb{G}}}$ is not pareto optimal); fix this $y$. Note that $g_{i}(\cdot)=g(i, \cdot)$. We will prove that $M$ cannot be $\varepsilon$-DP.

Let $z$ be such that $d_{\mathbb{G}}(y, z)=\Delta_{\mathbb{G}}(i, y)$ and $g_{i}(z) \neq g_{i}(y)$. Let $w$ be a neighbor of $z$ such that $d_{\mathbb{G}}(y, w)=\Delta_{\mathbb{G}}(i, y)-1$ and $b=g_{i}(w)=$ $g_{i}(y)$. Now, assume that $M \varepsilon$-DP. It follows that

$$
\begin{aligned}
P(M(w) \neq b) & \leq e^{\varepsilon d_{\mathscr{G}(y, w)}} P(M(y) \neq b) \\
& =e^{\varepsilon\left(\Delta_{\mathbb{G}}(i, y)-1\right)} P(M(y) \neq b)<1 /\left(1+e^{\varepsilon}\right)
\end{aligned}
$$

The First inequality is due to the DP constrains on $M$. The second inequality is due to the fact that $M$ is strictly better than $U_{\Delta_{\mathbb{G}}}$ on $y$ and the fact that $P\left(U_{\Delta_{\mathbb{G}}}(y) \neq g_{i}(y)\right)=e^{-\varepsilon\left(\Delta_{\mathbb{G}}(i, y)-1\right)} /\left(1+e^{\varepsilon}\right)$. Now if $M$ is $\varepsilon$-DP, then $P(M(z) \neq 1-b) \geq e^{-\varepsilon} P(M(w) \neq 1-$ $b)$, which together with (6) gives us $P\left(M(z) \neq g_{i}(z)\right)>1 /(1+$ $\left.e^{\varepsilon}\right)$; alternatively, $P\left(M(z)=g_{i}(z)\right)<e^{\varepsilon} /\left(1+e^{\varepsilon}\right)$. Since we know that $M$ is "better" than $U_{\Delta_{\mathbb{G}}}$, and in particular, $P\left(M(z)=g_{i}(z)\right) \geq$ $P\left(U_{\Delta_{\mathbb{G}}}(z)=g_{i}(z)\right)=e^{\varepsilon} /\left(1+e^{\varepsilon}\right)$, the above implies that $M$ is not $\varepsilon$-DP. Thus, we conclude the $U_{\Delta_{\mathbb{G}}}$ is pareto optimal.

\section{A.7 Proof of Lemma 4.5}

Lemma A.1. Arbitrarily fix a graph, $G$, that contains all the nodes and a subset of edges of the neighborhood graph, $\mathbb{G}$, and an $X \subseteq \mathcal{X}$. If $d_{G}$ is the shortest path length metric over $G$, then for every $x, y \in \mathcal{D}$,

$d_{G}(x, y) \geq d_{\mathbb{G}}(x, y)=|| x-y \|_{1} \geq \sum_{j \in X}\left|x_{j}-y_{j}\right| \geq\left|\sum_{j \in X}\left(x_{j}-y_{j}\right)\right|$.

Proof. Let $\mathbb{G}$ be the neighborhood graph over $\mathcal{D}$. Arbitrarily fix $G, d_{G}$, and $X$ as specified above (in the lemma). Since $G$ contains all the nodes and a subset of edges of $\mathbb{G}, d_{G}(x, y) \geq d_{\mathbb{G}}(x, y)$, where $d_{\mathbb{G}}$ is the shortest path length metric over $\mathbb{G}$. Furthermore, it is a simple observation that $d_{\mathbb{G}}$ is the same as $\ell_{1}$-metric over the databases (which follows from a simple induction argument). Hence, it follows that $d_{G}(x, y) \geq\|x-y\|_{1}$. The second inequality holds since $X \subseteq X$ and $\|x-y\|_{1}=\sum_{j \in \mathcal{X}}\left|x_{j}-y_{j}\right|$. The Third inequality follows from the reverse triangle inequality. This completes the proof. 
Proof of Lemma 4.5. Arbitrarily fix $\beta, k \geq 1$, and $r \geq 0$. Let $g$ be the $(\beta, r)$-anomaly identification function and $\lambda_{k}$ be as given by (3). Let $\Delta_{\mathbb{G}}$ and $\Delta_{G_{S}}$ be the mdd-functions for $g$, where $\mathbb{G}$ is neighborhood graph and $G_{S}$ is the $k$-sensitive neighborhood graph for $(\beta, r)$-anomaly. Next, arbitrarily fix a record $i$ and a node (database) $x$ in $G_{S}$.

We first show that $\Delta_{G_{S}}(i, x) \geq \lambda_{k}(i, x) \geq 1$. Below, we show that $\Delta_{G_{S}}(i, x) \geq \Delta_{\mathbb{G}}(i, x)$.

$$
\begin{aligned}
\Delta_{G_{S}}(i, x) & =\min _{y \in \mathcal{D}: g(i, x) \neq g(i, y)} d_{G_{S}}(x, y) \\
& \geq \min _{y \in \mathcal{D}: g(i, x) \neq g(i, y)} d_{\mathbb{G}}(x, y)=\Delta_{\mathbb{G}}(i, x)
\end{aligned}
$$

The first inequality follows from the fact that $G_{S}$ contains all the nodes and a subset of edges of $\mathbb{G}$. Hence, from Lemma 4.3, we conclude that if $B_{x}(i, r) \geq \beta+1-k$ then $\Delta_{G_{S}}(i, x) \geq \lambda_{k}(i, x) \geq 1$.

We now let $B_{x}(i, r)<\beta+1-k$ and $b=g(i, x)$. Here, it is clear that $\lambda_{k}(i, x) \geq 1$. Fix any $y$ in $G_{S}$ such that $g(i, y) \neq b$ and $\Delta_{G_{S}}(i, x)=d_{G_{S}}(x, y)$.

Consider the case of $x_{i}=0$. Here, it must hold that $y_{i} \geq 1$ and $B_{y}(i, r) \leq \beta$. Now, on any of the shortest path from $x$ to $y$, we will first reach a database $z$, where $i$ is $k$-sensitive, and hence, $B_{z}(i, r) \geq \beta+1-k$ (from Lemma A.2). Thus, for this $z$, we get

$$
\begin{aligned}
\Delta_{G_{S}}(i, x) & =d_{G_{S}}(x, z)+d_{G_{S}}(z, y) \\
& \geq d_{G_{S}}(x, z) \\
& \geq\left(B_{z}(i, r)-B_{x}(i, r)\right) \\
& \geq \beta+1-k-B_{x}(i, r)=\lambda_{k}(i, x) .
\end{aligned}
$$

The second inequality follows from Lemma $A .1$, and the third one follows because $B_{z}(i, r) \geq \beta+1-k$.

In the case, when $x_{i} \geq 1$, it must hold that either $y_{i}=0$ or $B_{y}(i, r) \geq \beta+1$. If $y_{i}=0$, then on any of the shortest path from $x$ to $y$, we will first reach a database $z$, where $i$ becomes $k$-sensitive, i.e., $B_{z}(i, r) \geq \beta+1-k$ (from Lemma A.2). If $z$ is the first such database, then $z_{i} \geq x_{i}$. Thus, we get the following.

$$
\begin{aligned}
d_{G_{S}}(x, y) & =d_{G_{S}}(x, z)+d_{G_{S}}(z, y) \\
& \geq\left(B_{z}(i, r)-B_{x}(i, r)\right)+\left|z_{i}-y_{i}\right| \\
& \geq 1+\beta-k-B_{x}(i, r)+x_{i} .
\end{aligned}
$$

The first inequality follows from Lemma A.1, and the second one follows from the fact that $B_{z}(i, r) \geq \beta+1-k$ and $x_{i} \leq z_{i}$. But if $B_{y}(i, r) \geq \beta+1$, then

$$
d_{G_{S}}(x, y)=d_{G_{S}}(x, y) \geq\left|B_{y}(i, r)-B_{x}(i, r)\right| \geq 1+\beta-B_{x}(i, r)
$$

From (7) and (8), we get the following, which is sufficient to establish that $\lambda_{k}$ is a lower bound on the $\Delta_{G_{S}}$.

$$
\Delta_{G_{S}}(i, x) \geq 1+\beta-B_{x}(i, r)+\min \left(0, x_{i}-k\right)=\lambda_{k}(i, x)
$$

Next, we show that $\lambda_{k}$ is 1-Lipschitz continuous. Fix an arbitrary neighbor, $y$, of $x$ such that $\lambda_{k}(i, x) \neq \lambda_{k}(i, y)$, otherwise, the continuity condition is trivially satisfied. If both $x$ and $y$ satisfy the first condition of $\lambda_{k}$, then the continuity condition is satisfied by Lemma 4.3. So assume that $x$ and $y$ satisfy the second condition of $\lambda_{k}$. Here, all the cases except for the following, trivially follow from the fact that $\|x-y\|_{1}=1$.

(a) $\lambda_{k}(i, x)=\beta+1-B_{x}(i, r)$ and $\lambda_{k}(i, y)=\beta+1-B_{y}(i, r)+y_{i}-k$ (b) $\lambda_{k}(i, x)=\beta+1-B_{x}(i, r)+x_{i}-k$ and $\lambda_{k}(i, y)=\beta+1-B_{y}(i, r)$ If $(a)$ holds then $(b)$ also does by symmetry (i.e., $\left|\lambda_{k}(i, x)-\lambda_{k}(i, y)\right|=$ $\left.\lambda_{k}(i, y)-\lambda_{k}(i, x) \mid\right)$ as $x$ and $y$ are picked arbitrarily. $(a)$ holds if $x_{i}-k \geq 0$ and $y_{i}-k \leq 0$; further, $\|x-y\|_{1}=1$ implies that $x_{i}-k=0$ and $-1 \leq y_{i}-k \leq 0$. When $y_{i}-k=0$, the continuity condition is satisfied as $\left|B_{x}(i, r)-B_{y}(i, r)\right| \leq 1$. However, $y_{i}-k=-1$ is not possible since $\|x-y\|_{1}=x_{i}-y_{i}=1$ implies that $i$ is $k$-sensitive with respect to $x$ or $y$, which implies that either $B_{x}(i, r)$ or $B_{y}(i, r)$ is at least $\beta+1-k$ (from Lemma A.2); this contradicts the assumption for this case. Hence, it follows that here the continuity condition is satisfied as well.

Lastly, consider the case, where $y$ and $x$ respectively satisfy the first and the second condition of $\lambda_{k}$-this is without loss of generality due to symmetry. This will be possible if $B_{x}(i, r)=\beta-k$ and $B_{y}(i, r)=\beta-k+1$. Thus, in all the subcases below, $x_{i} \leq y_{i} \leq$ $x_{i}+1$.

Consider the subcase of $x_{i}=0$. Here, $\lambda_{k}(i, x)=1$ and $y_{i}$ is either 0 or 1 . If $y_{i}=0$, then we have:

$\lambda_{k}(i, y)=\Delta_{\mathbb{G}}(i, y)=2$ for $k=1$, and $\lambda_{k}(i, y)=\Delta_{\mathbb{G}}(i, y)=1$ for $k>1$

But if $y_{i}=1, \lambda_{k}(i, y)=\Delta_{\mathbb{G}}(i, y)=\min \left(y_{i}, k\right)=1$ as $k \geq 1$. Hence, the continuity condition is satisfied for this subcase, when $x_{i}=0$.

Next, let $x_{i} \geq 1$; thus under this subcase it follows that

$$
\begin{aligned}
& \lambda_{k}(i, x)=1+k+\min \left(0, x_{i}-k\right)=1+\min \left(x_{i}, k\right) \\
& \lambda_{k}(i, y)=\Delta_{\mathbb{G}}(i, y)=\min \left(y_{i}, k\right) \quad\left(\text { since } x_{i} \leq y_{i}\right)
\end{aligned}
$$

Clearly, if $x_{i}<k$, then $\lambda_{k}(i, x)=1+x_{i}$ and $x_{i} \leq \lambda_{k}(i, y) \leq$ $x_{i}+1$; but if $x_{i} \geq k$, then $\lambda_{k}(i, x)=1+k$ and $\lambda_{k}(i, y)=k$ since $x_{i} \leq y_{i} \leq x_{i}+1$; hence the continuity condition is fulfilled in this subcase as well.

In all of the above case, $\left|\lambda_{k}(i, x)-\lambda_{k}(i, y)\right| \leq 1$. Since $\beta, k, r, i, x$, and $y$ (neighbor of $x$ ) were picker arbitrarily, we conclude that $\lambda_{k}$ is 1-Lipschitz continuous lower bond on the $\Delta_{G_{S}}$. This completes the proof.

\section{A.8 Proof of Theorem 4.7}

Proof. Fix any $k \geq 1, \varepsilon>0$, a valid $\varepsilon / 2$-differentially private mechanism, $M$, an anomaly identification query, $(i, g)$, and a nonnegative 2-Lipschitz continuous lower bound, $\delta$, on $\Delta_{G_{S}}-\Delta_{\mathbb{G}}$, where $\Delta_{G_{S}}$ and $\Delta_{\mathbb{G}}$ respectively correspond to the $k$-sensitive neighborhood graph for the anomaly definition corresponding to $g$, and the neighborhood graph. Let $U_{\delta}$ be the mechanism that Construction 2 yields. Next, fix arbitrary databases $x$ and $y$ that are neighbors in $G_{S}$.

When $\delta(i, x)=\delta(i, y)=0, \mathrm{P}\left(U_{\delta}(z)=b\right)=\mathrm{P}(M(z)=b)$ for every database $z$ and $b$ in $\{0,1\}$. The privacy constraints in this case, are trivially satisfied.

Next, consider the case, where $\delta(i, x)>\delta(i, y) \geq 0-$ this is without loss of generality as $x$ and $y$ are picked arbitrarily. Since $M$ is valid $\varepsilon / 2$-differentially private, we get the following for $g(i, x)=b$ for some $b \in\{0,1\}$,

$$
1-\mathrm{P}(M(x) \neq b) e^{-\varepsilon / 2} \leq e^{\varepsilon}(1-\mathrm{P}(M(y) \neq b))
$$

Recall that $G_{S}$ is a subgraph of $\mathbb{G}$ and contains a subset of edges of $\mathbb{G}$, and $\Delta_{\mathbb{G}}(i, z) \geq 1$ for every database $z$. Hence, it follows that $\Delta_{G_{S}}(i, z) \geq \Delta_{\mathbb{G}}(i, z) \geq 1$, and $\Delta_{G_{S}}(i, x)=1$ implies $\Delta_{\mathbb{G}}(i, x)=1$. Thus, from the above it follows that when $\Delta_{G_{S}}(i, x)-\Delta_{\mathbb{G}}(i, x) \geq$ 
$\delta(i, x)>0$, it must hold that $\Delta_{G_{S}}(i, x) \geq 2$. Since $d_{G_{S}}(x, y)=1$ and $\Delta_{G_{S}}(i, x) \geq 2$, we have $g(i, x)=g(i, y)$. So, let $b=g(i, x)$. From (9), we get the following.

$$
\begin{aligned}
& 1-\mathrm{P}(M(x) \neq b) e^{-\varepsilon / 2} \leq e^{\varepsilon}(1-\mathrm{P}(M(y) \neq b)) \\
\Longrightarrow & 1-e^{\varepsilon} \leq \mathrm{P}(M(x) \neq b) e^{-\varepsilon / 2}-\mathrm{P}(M(y) \neq b) e^{\varepsilon} \\
& \text { since } \delta \text { is 2-Lipschitz continuous, we get } \\
& 1-e^{\varepsilon} \leq \frac{\mathrm{P}(M(x) \neq b)}{e^{\frac{\varepsilon}{4}(\delta(i, x)-\delta(i, y))}}-\mathrm{P}(M(y) \neq b) e^{\varepsilon} \\
& \text { since LHS is negative, and } \delta \geq 0, \text { the following holds } \\
& 1-e^{\varepsilon} \leq e^{-\frac{\varepsilon}{4} \delta(i, y)}\left(\frac{\mathrm{P}(M(x) \neq b)}{e^{\frac{\varepsilon}{4}(\delta(i, x)-\delta(i, y))}}-\mathrm{P}(M(y) \neq b) e^{\varepsilon}\right) \\
\Longrightarrow & 1-\frac{\mathrm{P}(M(x) \neq b)}{e^{\frac{\varepsilon}{4} \delta(i, x)} \leq e^{\varepsilon}\left(1-\frac{\mathrm{P}(M(y) \neq b)}{e^{\frac{\varepsilon}{4} \delta(i, y)}}\right)} \\
\Longrightarrow & \mathrm{P}\left(U_{\delta}(x)=b\right) \leq e^{\varepsilon} \mathrm{P}\left(U_{\delta}(y)=b\right)
\end{aligned}
$$

In a similar fashion, by swapping $x$ and $y$ in (9), one can show that the privacy constraint $\mathrm{P}\left(U_{\delta}(y)=b\right) \leq e^{\varepsilon} \mathrm{P}\left(U_{\delta}(x)=b\right)$ also holds. Below we show that the other constraints are also satisfied.

$$
\frac{\mathrm{P}\left(U_{\delta}(x) \neq b\right)}{\mathrm{P}\left(U_{\delta}(y) \neq b\right)}=\frac{\mathrm{P}(M(x) \neq b) e^{-\frac{\varepsilon}{4} \delta(i, x)}}{\mathrm{P}(M(y) \neq b) e^{-\frac{\varepsilon}{4} \delta(i, y)}} \leq e^{\varepsilon}
$$

The above inequality holds because $M$ is $\varepsilon / 2$-DP and $\delta$ is 2-Lipschitz continuous.

Since all the privacy constraints hold for arbitrarily picked neighbors and $\delta$ (which satisfies the conditions specified in the claim), and a valid $\varepsilon / 2$-differentially private $M$ for an anomaly identification query, the claim holds in general.

As for the claim of accuracy, it is a direct implication from the Construction 2. This completes the proof.

\section{A.9 Composition}

Here, we assume that every mechanism has its independent source of randomness and has the domain $\mathcal{D}$. Further, $E(G)$ for a graph $G$ denotes the set of edges in $G$. We make the following very simple observation.

ObServation 1. For any simple graphs $G$ and $G^{\prime}$ over $\mathcal{D}$, two databases are neighbors in the graph $H=\left(\mathcal{D}, E(G) \cap E\left(G^{\prime}\right)\right)$ if and only if they are neighbors in $G$ and $G^{\prime}$.

Claim 3. If mechanisms $M_{1}$ and $M_{2}$ are respectively $\varepsilon_{1}-S P$ for $G_{S_{1}}$ and $\varepsilon_{2}-S P$ for $G_{S_{2}}$, then $M(x):=\left(M_{1}(x), M_{2}(x)\right)$ for every $x$ is $\left(\varepsilon_{1}+\varepsilon_{2}\right)-S P$ for $G_{S}=\left(\mathcal{D}, E\left(G_{S_{1}}\right) \cap E\left(G_{S_{2}}\right)\right)$.

Proof Sketch. The claim follows from $M_{1}$ and $M_{2}$ being SP for $\varepsilon_{1}$ and $\varepsilon_{2}$, and Observation 1, which ensures that the privacy constraints will be met for neighbors in $G_{S}$.

We say, for $Y \subseteq \mathcal{X}$, a mechanism $M$ is $Y$-dependent if and only if for every $r \in \operatorname{Range}(M)$ and $x$ and $y$ such that $x_{i}=y_{i}$ for every $i \in Y, P(M(x)=r)=P(M(y)=r)$.

ClaIM 4. For any partition of $\mathcal{X}=Y_{1} \sqcup Y_{2}$, if mechanisms $M_{1}$ and $M_{2}$ are respectively $Y_{1}$-dependent $\varepsilon_{1}-S P$ for $G_{S_{1}}$ and $Y_{2}$-dependent $\varepsilon_{2}$ $S P$ for $G_{S_{2}}$, then $M(x):=\left(M_{1}(x), M_{2}(x)\right)$ for every $x$ is $\max \left(\varepsilon_{1}, \varepsilon_{2}\right)$ $S P$ for $G_{S}=\left(\mathcal{D}, E\left(G_{S_{1}}\right) \cap E\left(G_{S_{2}}\right)\right)$.
Proof SKeTCH. Firstly, note that $M_{1}$ and $M_{2}$ being SP for $\varepsilon_{1}$ and $\varepsilon_{2}$ along with Observation 1, ensure that the privacy constraints will be met for neighbors in $G_{S}$ for some value of $\varepsilon$. Further, since every neighbor in $G_{S}$ differ by one record and mechanisms $M_{1}$ and $M_{2}$ are respectively $Y_{1}$ and $Y_{2}$ dependent (for an arbitrarily fixed partition), every privacy constraint will hold for either $\varepsilon_{1}$ or $\varepsilon_{2}$. From here the claim follows.

\section{A.9.1 Proof of Claim 5.}

Lemma A.2. Fix arbitrary values for $k \geq 1, \beta \geq 1$ and $r \geq 0$. For $(\beta, r)$-anomaly, for every record $i \in \mathcal{X}$ and every database $x \in \mathcal{D}$,

$i$ is $k$-sensitive with respect to $x \Longleftrightarrow B_{x}(i, r) \geq \beta+1-k$.

Proof. Arbitrarily fix $k, \beta \geq 1, r \geq 0, i \in \mathcal{X}$, and $x \in \mathcal{D}$. Further, fix $p$ to be the normality property corresponding to $(\beta, r)$-anomaly.

Firstly, we prove the "if" direction through its contrapositive. So assume $B_{x}(i, r)<\beta+1-k$. Now, for every database $y$ such that $\|x-y\|_{1} \leq k, B_{y}(i, r) \leq \beta$ as we can only add up to $k$ records in $x$. Thus for each of the above $y, p(i, y)=0$, which follows from the definition of $(\beta, r)$-anomaly, and $i$ is not $k$-sensitive with respect to $x$. This completes the proof for "if" direction.

Next, we prove the "only if" direction. Let $B_{x}(i, r) \geq \beta+1-k$. Now, obtain a database $y$ by adding $k$ records that are the same as $i$ to $x$. For this $y$, it holds that $\|x-y\|_{1}=k$ and $p(i, y)=0$ because $y_{i} \geq 1$ and $B_{y}(i, r) \geq \beta+1$ (since $k \geq 1$ ). Hence, we conclude that $i$ is $k$-sensitive with respect to $x$. And this completes the proof as $k, \beta, r, i$, and $x$ were chosen arbitrarily.

For any $i \in \mathcal{X}$ and $r \geq 0$, we write $Y(i, r)$ to denote the set $\{j \in \mathcal{X}: d(i, j) \leq r\}$.

Claim 5. For any given $n \in \mathbb{N}$ and every $t=1, \ldots, n$, arbitrarily fix $\varepsilon_{t}, r_{t}>0, k_{t}, \beta_{t} \geq 1$, and a mechanism, $M_{t}: \mathcal{D} \rightarrow\{0,1\}$, that is $\varepsilon_{t}-S P$ for $k_{t}$-sensitive neighborhood graph corresponding to $\left(\beta_{t}, r_{t}\right)$-anomaly and is also $Y\left(i_{t}, r_{t}\right)$-dependent. Further, let $m$ be the maximum number of $i_{t}$ 's that are within any ball of radius $\max \left(r_{1}, \ldots, r_{n}\right), \varepsilon=\max \left(\varepsilon_{1}, \ldots, \varepsilon_{n}\right), k=\min \left(k_{1}, \ldots, k_{n}\right), \beta=$ $\max \left(\beta_{1}, \ldots, \beta_{n}\right)$, and $r=\min \left(r_{1}, \ldots, r_{n}\right)$.

If $M(x):=\left(M_{1}(x), \ldots, M_{n}(x)\right)$ for every $x$, then $M$ is $m \varepsilon$-sensitively private for $k$-sensitive neighborhood graph corresponding to $(\beta, r)$ anomaly.

Proof. Arbitrarily fix the values for all the symbols used in the claim above as per the specification.

Firstly, we consider the guarantee with respect to the sensitive neighborhood graph. Here it is sufficient to show that the $k$ sensitive neighborhood graph, $G_{S}$, corresponding to $(\beta, r)$-anomaly, is a subgraph of the $k_{t}$-sensitive neighborhood graph, $G_{S}^{t}$, corresponding to $\left(\beta_{t}, r_{t}\right)$-anomaly for every $t$. Thus we show that, for any $t$ and two databases $x$ and $y$, if $x$ and $y$ are neighbors in $G_{S}$, then they are neighbors in $G_{S}^{t}$. So arbitrarily fix $x$ and $y$ that are neighbors in $G_{S}$ and $t \in[n]$. Since $x$ and $y$ are neighbors in $G_{S}$, there exists a record $i$ that $k$-sensitive with respect to $x$ or $y$. Let $i$ be $k$-sensitive with respect to $x$-this is without loss of generality since $x$ and $y$ are picked arbitrarily. Now, from Lemma A.2, we get that $B_{x}(i, r) \geq \beta-k+1$. Since $\beta \geq \beta_{t}$ and $k \leq k_{t}, B_{x}(i, r) \geq \beta_{t}-k_{t}+1$; this implies that $i$ is $k_{t}$-sensitive with respect to $x$ (Lemma A.2), and thus, $x$ and $y$ are neighbors in $G_{S}^{t}$. Hence, we conclude that $G_{S}$ is a subgraph of every $G_{S}^{t}$. 
Next, we prove the bound on the divergence of probabilities to show that the loss in privacy is at max $m \varepsilon$. For any $i \in \mathcal{X}$, let $A_{i}$ be such that for every $t \in[n], t \in A_{i} \Longleftrightarrow d\left(i, i_{t}\right) \leq r^{\prime}$, where $r^{\prime}=\max \left(r_{1}, \ldots, r_{n}\right)$. And let $m=\max _{i \in X}\left|A_{i}\right|$. Arbitrarily fix, the neighboring databases $x$ and $y$ in $G_{S}$ and $w \in\{0,1\}^{n}$. Let $i$ be the record in which $x$ and $y$ differ. Now it follows that

$$
\begin{aligned}
\frac{\mathrm{P}(M(x)=w)}{\mathrm{P}(M(y)=w)} & =\prod_{t \in A_{i}} \frac{\mathrm{P}\left(M_{r}(x)=w_{r}\right)}{\mathrm{P}\left(M_{r}(y)=w_{r}\right)} \times \prod_{l \in[n] \backslash A_{i}} \frac{\mathrm{P}\left(M_{l}(x)=w_{l}\right)}{\mathrm{P}\left(M_{l}(y)=w_{l}\right)} \\
& =\prod_{t \in A_{i}} \frac{\mathrm{P}\left(M_{t}(x)=w_{t}\right)}{\mathrm{P}\left(M_{t}(y)=w_{t}\right)} \leq \exp \left(\sum_{t \in A_{i}} \varepsilon_{t}\right) \leq \exp (m \varepsilon)
\end{aligned}
$$

Above, the first equality holds because each of the $M_{t}$ has its independent source of randomness. The second equality holds because each $M_{t}$ is $Y\left(i_{t}, r_{t}\right)$-dependent in addition to its randomness and $r_{t} \leq r^{\prime}$. The first inequality follows from $M_{t}$ being $\varepsilon_{t}$-SP for $G_{S}$, which is a subgraph of $G_{S}^{t}$. The last inequality follows from the fact that $\varepsilon \geq \varepsilon_{t}$ and $m \geq\left|A_{i}\right|$.

Lastly, note that for any $W \subseteq\{0,1\}^{n}$, it follows that

$$
\frac{\mathrm{P}(M(x) \in W)}{\mathrm{P}(M(y) \in W)} \leq \frac{\sum_{w \in W} \mathrm{P}(M(x)=w)}{\sum_{w \in W} \mathrm{P}(M(y)=w)} \leq \exp (m \varepsilon)
$$

Thus, we conclude that the claim holds.

\section{A.10 Relation of SP to other definitions}

A.10.1 One-sided differential privacy (OSDP) [13]. It allows for mechanisms to be private that can reveal the presence or absence of a sensitive record in the database. We explain this below. Consider two neighboring databases $x$ and $y$ (i.e., they differ by one record) such that $x$ has exactly one sensitive record and $y$ has no sensitive record, and an $\varepsilon$-OSDP mechanism $M: \mathcal{D} \rightarrow\{0,1\}$ with $P(M(x)=$ $0)=0$ and $P(M(y)=0)=1-$ note this is possible as $M$ only needs to satisfy $P(M(x) \in b) \leq e^{\varepsilon} P(M(y) \in b)$ for $b \in\{0,1\}$. Now, if we pick $x$ or $y$ randomly and reveal the output of $M$, the output will reveal which database was used, and hence if the sensitive record was present or not.

A.10.2 Tailored differential privacy (TDP) [36]. SP is a special case of TDP. Which becomes clearer once we restate TDP for the unbounded case, which we deal with. For $\alpha: \mathcal{X} \times \mathcal{D} \rightarrow \mathbb{R}_{\geq 0}$, a mechanism is $\alpha(\cdot)$-TDP if for every two databases, $x$ and $y$ differing in a record $i$, and every $R \subseteq \operatorname{Range}(M), P(M(x) \in R) \leq$ $e^{\alpha(i, x)} P(M(y) \in R)$. Let for every $i$ and $x, \alpha(i, x)=\varepsilon d_{G_{S}}\left(x, x^{\prime}\right)$ $\left(x_{j}^{\prime}=x_{j}\right.$ for every $j \neq i$ and $\left.x_{i}-x_{i}^{\prime}=1\right)$. Now, it is immediate that a mechanism is $\alpha(\cdot)$-TDP if and only if it is $\varepsilon$-SP for $G_{S}$. A similar statement holds true for Blowfish privacy [25], which follows by considering the sensitive neighborhood graph to be the policy graph. 